\title{
Lusioersily
}

\section{Semi-monolayer covering rough set on set-valued information systems and its efficient computation}

Wu, Z., Wang, H., Chen, N., \& Luo, J. (2021). Semi-monolayer covering rough set on set-valued information systems and its efficient computation. International Journal of Approximate Reasoning, 130, 83-106. https://doi.org/10.1016/j.jjar.2020.12.011

Link to publication record in Ulster University Research Portal

\section{Published in:}

International Journal of Approximate Reasoning

Publication Status:

Published (in print/issue): 31/03/2021

DOI:

10.1016/j.jjar.2020.12.011

\section{Document Version}

Author Accepted version

\section{General rights}

Copyright for the publications made accessible via Ulster University's Research Portal is retained by the author(s) and / or other copyright owners and it is a condition of accessing these publications that users recognise and abide by the legal requirements associated with these rights.

\section{Take down policy}

The Research Portal is Ulster University's institutional repository that provides access to Ulster's research outputs. Every effort has been made to ensure that content in the Research Portal does not infringe any person's rights, or applicable UK laws. If you discover content in the Research Portal that you believe breaches copyright or violates any law, please contact pure-support@ulster.ac.uk. 


\title{
Semi-Monolayer Covering Rough Set on Set-valued Information Systems and Its Efficient Computation
}

\author{
Zhengjiang $\mathrm{Wu}^{\mathrm{a}}$, Hui Wang ${ }^{\mathrm{b}}$, Ning $\mathrm{Chen}^{\mathrm{a}}$, Junwei $\mathrm{Luo}^{\mathrm{a}}$ \\ ${ }^{a}$ College of Computer Science and Technology, Henan Polytechnic University, Jiaozuo, China 454003 \\ ${ }^{b}$ School of Computing, University of Ulster at Jordanstown, Newtownabbey BT37 OQB, Northern Ireland, UK
}

\begin{abstract}
For set-valued information systems, there are many original dot-based approximation models based on tolerance relations and other developed tolerance relations. Because of the lack of efficient algorithms, they are not to accommodate the bigger and bigger set-valued information table. Therefore, it is a real challenge on how to efficiently calculate a high-quality approximation set in set-valued information systems. To address the challenge, we propose reliable approximation operators based on semi-monolayer covering for set-valued information systems. Benefiting from considerable research about tolerance rough set models and covering rough set models, the proposed approximation operators used a piecewise design to effectively reduce the negative effects of the set-valued records and provided high-quality approximation sets for set-valued information systems. Furthermore, the reliable semi-monolayer covering approximation sets are more easily granulated and efficiently calculated than before. Based on the equivalent granule-based forms, the corresponding granular algorithms are designed for the the improved approximation sets. The experiments on some UCI data sets show the improved approximation sets are high quality and efficient computational in set-valued information systems.
\end{abstract}

Keywords: set-valued information system, semi-monolayer covering, approximation quality, granule-based form of approximation operator

\section{Introduction}

Rough set theory [?] is a mathematical tool to deal with knowledge in information systems. A high-quality approximation set is the foundation of effective rule acquisition and attribute reduction. It is a real challenge on how to efficiently get high-quality approximation sets in an information system.

Set-valued information systems are the generalized model of single-valued information systems. In set-valued information systems, some records have more than one value for an attribute. There exist two kinds of semantic interpretation for the set value in the system: conjunctive interpretation and disjunctive interpretation. Incomplete information systems can usually be considered as disjunctively interpreted set-valued information systems [? ], in which the missing value can be considered as the set of all possible values for the attribute [? ? ? ? ]. Therefore, when we discussed the rough set models on set-valued information systems, the ones used for incomplete information systems should be taken into account.

\subsection{Approximation Quality of Approximation Set}

The approximation operator is the core definition of rough set model. The approximation space, basic algebra, and operator form are all primitive notions consisting of approximation operators, as shown in Figure 1. The approximation space herein is a tuple consisting of a universe and a binary relation on it, or a universe and a covering on it [? ? ? ]. Any change in those factors will affect the approximation quality and other properties of the approximation operator

Email addresses: wuzhengjiang@hpu. edu.cn (Zhengjiang Wu), h. wang@ulster. ac. uk (Hui Wang), nchen@hpu . edu . cn (Ning Chen), luojunwei@hpu.edu.cn (Junwei Luo) 


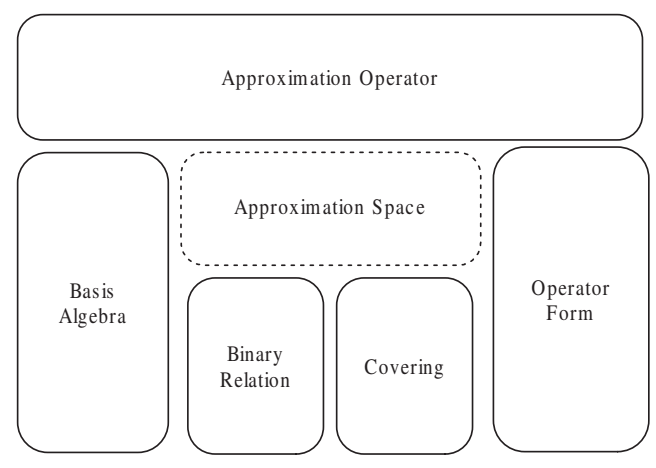

Figure 1: Important Factors for Approximation Operator

[? ? ]. For example, the development from fuzzy rough set [?] to $(\mathscr{S}, \mathscr{T})$-fuzzy rough set [? ] is an evolution of basic algebra; the multiple rough set models [? ? ? based on covering approximation space have different operator forms. Of course, most of the development of rough set models are about approximation space, such as Pawlak rough set model [? ], variable precision rough set [? ? ], covering rough set [? ], dominance relation rough set [? ] and etc.

For set-valued (incomplete) information systems, considerable research has been conducted to develop the tolerance relation and basic algebra of the rough set model. In the tolerance relation presented by M. Kryszkiewicz [? ], the key point is to assign a null value to all missing attribute values of an incomplete information table [? ]. The tolerance relation is easy to understand. Therefore, some researchers still use this model even today [? ? ?. However, the improvement of the rough models based on tolerance relation is never stopped.Stefanowski and Tsoukias [?] proposed non-symmetric tolerance relation to improve the approximation quality of tolerance rough sets in incomplete information systems. In the model, a non-symmetric tolerance relation replaces an original tolerance relation to limit set-valued records access to the tolerance neighborhood. Wang [? ] proposed a limited tolerance relation to avoid too many set-valued records involved in the tolerance neighborhood by adding an extraordinary condition. Nguyen [?] proposed the extended tolerance relation based on tolerance degrees on incomplete information systems. Under the different thresholds, the extended tolerance relation can degrade to tolerance, limit tolerance, and equivalence relations. Dai and Tian [? ? ] define a fuzzy similar relation and construct a fuzzy rough set model for set-valued information systems. Wei [?] generalized the rough fuzzy set models on set-valued information systems. Ahmed [? ] redefined the fuzzy tolerance relation on fuzzy set-valued information systems. Whatever by optimizing tolerance relation or updating basic algebra, the central idea is to highlight single-valued records and restrict set-valued records in approximation sets.

Covering is another way to construct approximation space on set-valued information systems. The block in covering is a natural granule. Those granule-based forms can essentially improve the computation speed of approximation sets [? ? ? ]. Couso and Dubois [? ] had used a covering and its corresponding generalized rough set to describe the incomplete information. Wu and Yang [? ] had defined the complete coverings based on the tolerance relation in incomplete information systems. Leung and $\mathrm{Li}[?]$ proposed a maximal consistent block to acquire rules from incomplete information systems. Guan and Wang [? ] generalized non-symmetric tolerance relations in set-valued information systems, and proposed the maximal consistent blocks, and defined the A-approximation and Eapproximation based on maximal consistent covering approximation space. In turn, Yang [?] also used the maximal consistent block to improve the approximation quality of the neighborhood rough set in the incomplete information system. Based on general covering rough models, $\mathrm{Wu}$ and Chen [?] proposed semi-monolayer covering rough set. As a special covering, there exists a one-by-one correspondence between the record in set-valued information system and element in a semi-monolayer covering. Semi-monolayer covering can be considered as another interpretation of maximal consistent blocks set in the aspect of covering. In [? ], Guan and Wang have been more concerned with the reduction of set-valued information systems, but in [? ], Wu and Chen aim to improve the computability of the approximation sets. However, single-valued records and set-valued records have not been effectively distinguished in the approximation operators, as shown at the tolerance optimization. Therefore, it still has much room to improve the approximation quality of approximation set in set-valued information systems. With a similar idea in the develop- 
ment of tolerance relations, it need to enhance the influence from more valuable single-valued records or weaken the influence from secondary set-valued ones in approximation sets.

In covering rough set models, it is a common way to optimize the approximation quality by improving the operator's form. For example, the four types of covering-based rough sets by W. Zhu [? ? ], the covering approximation operators listed in [?]. In essence, the optimization of the operator's form is the optimization of the rules of selecting the elements for the final approximation sets, and it is a practical solution to improve approximation quality. Specific to covering rough set models in set-valued information systems, we can optimize the operator's form to differentiate valuable single-valued records from set-valued records in approximation operators. In the new approximation operator, the single-valued block replaces the whole block to involve in the judgment of the relationship with $X$, but the set-valued records are treated as dependent on the single-valued records. After selecting which single-valued records are in the approximation set, the attached controversial records are appended in the final approximation sets. In this way, we distinguish the single-valued records from the set-valued records in the new approximation sets. The new approximation sets are closer to the approximated set than before.

\subsection{Efficient Computation of Approximation Set}

In rough set theory, the calculating approximation set is an unavailable step in knowledge representation and reduction. An efficient algorithm can contribute positively to expanding the application of rough set in data mining. Transforming the approximation sets into the granule-based form is an original but efficient approach, in which the approximation sets can be built with information granule piece by piece. More importantly, the cost of generating an equivalent block is far less than the cost of generating a neighborhood of one element in large-scale datasets [? ? ? ]. The granule-based form is not the only way to accelerate the calculation. By more efficient strategies and more powerful hardware, efficiently computing approximation sets were achieved by the incremental computation [? ? ? ? ? ? ? ? ? , the parallel computation [? ? ], the matrix representation of approximation set, the localization of the neighborhood [? ? ] or the combination of them, such as the parallel incremental approximation for approximation set [? ], the incremental strategy for the matrix representation of approximation sets in [? ? ], a parallel method for computing approximations based on matrix [? ? ]. However, once the approximation operators have an equivalent granule-based form, all of the above acceleration methods can be improved again. In this paper, the equivalent granulebased forms for the new approximation sets are proposed in SMC approximation space. Furthermore, according to the set-valued information system, the granular expression of approximation sets is processed for the information cells. The corresponding algorithms based on the granule expression are designed to accelerate the calculation of approximation sets.

The rest of this paper is organized as follows. In section 2, we recall some necessary definitions about set-valued information system and semi-monolayer covering, and give an approach to construct a semi-monolayer covering on the complete set-valued information system. In section 3, we propose the definition reliable semi-monolayer covering approximation operators (DE0, DA0, and GC0), and prove some properties of those approximation operators. In section 4, through a couple of theorems and corollaries, we analyzed the advantages and disadvantages of DE0 and DA0 approximation sets brought about by the new operator's form. The results have shown that the DE0 and DA0 approximation sets will closer to the approximated set $X$ than before. The weak monotonicity is become the cost of the improved operator's form. Section 5 is exactly to serve for the efficient computation of reliable covering approximation sets on set-valued information system. In this part, a series of granular algorithms (GAS) for DE0 and DA0 are designed, and the equivalence granule-based form of DE0 and DA0 provides a theoretic guarantee for the reliability of those granular algorithms. In section 6 , some experiments on real data sets verify the approximation quality of DE0 and DA0 and the efficiency of the granular algorithm in practice. The last part of this paper summarized this paper's content, innovation and insufficiency.

\section{Set-valued Information System and Semi-monolayer Covering on It}

Set-valued information system (abbr. SVIS) [? ] is a quadruple $(U, A, V, f)$, where $U$, which is called an universe, is a non-empty finite set of objects; $C$ is a finite set of attributes; Specially, $(U, A \cup\{d\}, V, f)$ is a set-valued decision information system. $A$ is condition attributes, and $d$ is a decision attribute with $A \cap\{d\}=\emptyset ; V=V_{A} \cup V_{d}$, where $V_{A}$ is the set of condition attribute values, $V_{d}$ is the set of decision attribute values; $f$ is a mapping from $U \times(A \cup\{d\})$ to $V$ satisfying that $f: U \times A \rightarrow 2^{V_{A}}$ is a set-valued mapping, and $f: U \times\{d\} \rightarrow V_{d}$ is a single-valued mapping. 
Definition 1. Let $S=(U, A, V, f)$ be a set-valued information system (abbr. SVIS), where $A=\left\{a_{1}, a_{2}, \ldots, a_{n}\right\}$. The information explanation of an object $x \in U$ in $S$ is a vector.

$$
\vec{x}=<f\left(x, a_{1}\right), f\left(x, a_{2}\right), \ldots, f\left(x, a_{n}\right)>=<\left\{x_{a_{1}}^{0}, x_{a_{1}}^{1}, \ldots, x_{a_{1}}^{m_{1}}\right\},\left\{x_{a_{2}}^{0}, x_{a_{2}}^{1}, \ldots x_{a_{n}}^{m_{2}}\right\}, \ldots,\left\{x_{a_{n}}^{0}, x_{a_{n}}^{1}, \ldots, x_{a_{n}}^{m_{n}}\right\}>
$$

Definition 2. [? ? ] Let $U$ be an universe and $C=\left\{K_{1}, K_{2}, \ldots, K_{n}\right\}$ be a family of subsets of $U$. If $\emptyset \notin C$ and $\cup C=U$, $C$ is called a covering on $U$. Every $K$ in $C$ is called a block in the covering. The description of an element $x$ in $C$ is $K_{x}=\{K \in C \mid x \in K\}$.

Definition 3. [? ? ] Let $C$ be a covering on $U$. An element $x$ belongs to $K \in C$, satisfying the condition $\forall S \in C(x \in$ $S \Rightarrow K \subseteq S$ ), is called a representative element of the block $K$. $C$ is called representative iff $\forall K \in C$, there exists $x \in K \forall S \in C(x \in S \Rightarrow K \subseteq S)$.

Representative elements are special ones in covering. Every block in representative covering has one representative element at least [? ? ]. However, the label "representative element" is local. For example, a representative element (the black-and-white point) in block $\mathrm{A}$ is not the representative one in block B in Figure 2(a). A one-by-one correspondence relation can not be established among the single-valued records in a set-valued information system and representative elements in a representative covering, just because of lack of global uniqueness. Otherwise, we can not guarantee that only single-valued records are allowed to participate in the computation of approximation sets in Definition 8.

Definition 4. [?] Let $U$ be an universe, and $C$ be a representative covering on $U$. If every $K \in C$ is indispensable, i.e. $\cup\left\{K^{\prime} \mid K^{\prime} \in C, K^{\prime} \neq K\right\} \subset U, C=\left\{K_{1}, K_{2}, \ldots, K_{n}\right\}$ is a semi-monolayer covering on $U$ (abbr. SMC).

- $x$ is a reliable element of $K$, if $\forall S \in C(x \in S \Rightarrow K=S)$. The set of all reliable elements in $U$ is $U_{0}$.

- $K_{0}$ is the reliable set of $K$, which consists of all reliable elements in $K$.

- $x$ is a controversial element, if $\exists K_{1}, K_{2} \in C, x \in K_{1}$ and $x \in K_{2}$.

Note: In this paper, the reliable set of $K_{i} \in C$ will be denoted as $K_{i 0}$ and $U_{0}=\cup\left\{K_{i 0} \mid K_{i} \in C\right\}$.

Therefore, we need a more special representative covering which can be isomorphic to the set-valued information table or its subsystem. As a special representative covering, there does not exist any nested relation between blocks in semi-monolayer covering. Every element in semi-monolayer covering has its unique label - the reliable element or controversial element [? ]. For example, the black points in Figure 2(b) are reliable elements, and the white point is a controversial element. Furthermore, maximal consistent blocks [? ? ] are the neighborhoods based on the generalized non-symmetric tolerance relation. If every block has more than one reliable element, those blocks consist of a semi-monolayer covering on the set-valued information system.

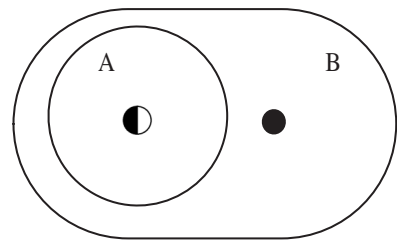

(a) Representative Covering

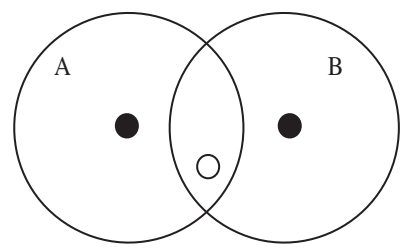

(b) Semi-monolayer Covering

Figure 2: Semi-monolayer Covering and General Representative Covering on SVIS

Theorem 1. Let $S=(U, A, V, f)$ be SVIS. A single-valued record $x \in U$ corresponds to a reliable element $x \in K$ in semi-monolayer covering $C$, where $K=\{y \mid \forall a \in A, f(x, a) \subseteq f(y, a)\}$.

Proof. If $x$ is a single-valued record, $x \in K$ and $K=\{y \mid \forall a \in A, f(x, a) \subseteq f(y, a)\}$. $K$ is the only one block containing $x$. Thus, $C$ is representative and $x \notin\left(\cup_{K^{\prime} \in C} K^{\prime}-K\right)$. Furthermore, $C$ is a semi-monolayer covering. 
Definition 5. Let $S=(U, A, V, f)$ be SVIS. For every set-valued record $y$, there exists a single-valued record $x$ satisfying that $\forall a \in A, f(x, a) \subseteq f(y, a)$. $S$ is called complete set-valued information system (abbr. CSVIS).

Actually, if $S=(U, A, V, f)$ be CSVIS, there exists an onto mapping from SVIS to SMC. In this mapping, a single-value record corresponds to a reliable element, a set-valued record corresponds to a controversial element, and every set-valued record has a related single-valued record on $U$ at least, such as the SVIS in Example 1.

Example 1. A journal editor invites two reviewers to evaluate 17 papers $U=\left\{x_{1}, x_{2}, \ldots, x_{17}\right\}$ in two aspects $A=$ $\{$ LinguisticQuality, Originality\}.Combined with the evaluation results from the two reviewers, the editor gets a setvalued information table. The results are shown in Table 1.

\begin{tabular}{ccc|ccc}
\hline & Linguistic Quality & Originality & & Linguistic Quality & Originality \\
\hline$x_{1}$ & Good & Good & $x_{2}$ & Good & Good \\
$x_{3}$ & Good & Good & $x_{4}$ & \{Good, Poor & Good \\
$x_{5}$ & \{Good, Poor & Good & $x_{6}$ & Poor & Good \\
$x_{7}$ & Poor & Good & $x_{8}$ & Poor & Good \\
$x_{9}$ & Good & $\{$ Good, Poor & $x_{10}$ & Good & $\{$ Good, Poor \\
$x_{11}$ & $\{$ Good, Poor & \{Good, Poor & $x_{12}$ & Poor & $\{$ Good, Poor \\
$x_{13}$ & Poor & \{Good, Poor $\}$ & $x_{14}$ & Good & Poor \\
$x_{15}$ & Good & Poor & $x_{16}$ & \{Good, Poor & Poor \\
$x_{17}$ & Poor & Poor & & & \\
\hline
\end{tabular}

Table 1: A Set-Valued Information System

The single-valued records are $x_{1}, x_{2}, x_{3}, x_{6}, x_{7}, x_{8}, x_{14}, x_{15}$, and $x_{17} . C=\left\{K_{1}, K_{2}, K_{3}, K_{4}\right\}$ is semi-monolayer covering on $U=\left\{x_{1}, x_{2}, \ldots, x_{17}\right\}$.

$$
\begin{gathered}
K_{1}=\left\{x_{1}, x_{2}, x_{3}, x_{4}, x_{5}, x_{9}, x_{10}, x_{11}\right\}, K_{2}=\left\{x_{6}, x_{7}, x_{8}, x_{4}, x_{5}, x_{11}, x_{12}, x_{13}\right\}, \\
K_{3}=\left\{x_{14}, x_{15}, x_{16}, x_{9}, x_{10}, x_{11}\right\}, K_{4}=\left\{x_{17}, x_{12}, x_{13}, x_{16}, x_{11}\right\} .
\end{gathered}
$$

$K_{10}=\left\{x_{1}, x_{2}, x_{3}\right\}, K_{20}=\left\{x_{6}, x_{7}, x_{8}\right\}, K_{30}=\left\{x_{14}, x_{15}\right\}$ and $K_{40}=\left\{x_{17}\right\}$ are the reliable sets of $K_{1}, K_{2}, K_{3}$ and $K_{4}$ correspondingly. Moreover, the set-valued information system in Table 1 is complete.

\section{The Definition of Reliable Semi-monolayer Covering Approximation Operators and Their Basic Properties}

In rough set theory, lower and upper approximation sets are used to approximate uncertain information in approximation space. How to obtain an approximation set as close as possible to the approximated set $X$ is a core problem for rough set operators. The existing approximation operators, approximation operator based on maximal consistent blocks [? ] and approximation operators based on semi-monolayer covering [? ], are unable to distinguish the reliable and controversial element on it. Therefore, we propose a new operator form to improve the approximation quality of approximation sets on set-valued information systems, and then we will discuss the advantages and disadvantages of the improvement of the operator's form in theory.

Definition 6. [? ] Let $U$ be an universe and $C$ be $S M C$ on $U$. The pair $(U, C)$ is called a SMC approximation space.

In [? ], Wu and Chen defined a series of semi-monolayer covering approximation operators (DE, DA, and GC). $\mathrm{DE}$ and DA can be considered as the specific covering rough approximation operators on SMC approximation space. Specifically, GC corresponds to $\underline{C}$ and $\bar{C}$ in [? ]; the upper DE approximation operator corresponds to $\bar{C}$ and the lower DA approximation operator corresponds to $C$ in [? ? ? ]; lower DE, upper DE, lower DA, and upper DA approximation operators correspond to $\mathrm{apr}^{\prime}, \overline{a p r}^{\prime \prime}, \overline{\mathrm{apr}^{\prime \prime}}{ }_{c}, \overline{\mathrm{apr}^{\prime}}{ }_{c}$ in [? ]. On another hand, if every maximal consistent block contains one single-valued record at least, those blocks will form a semi-monolayer covering on setvalued information systems. E-approximation and A-approximation operators based on maximal tolerance blocks [? ] can be viewed as DE and DA on the complete set-valued information system.

Definition 7. [? ? ] Let $(U, C)$ be a $S M C$ approximation space and $X \subseteq U$, the semi-monolayer covering approximation operators (abbr. SMC-APPS), namely DE, DA and GC, are defined as follows: 
(DE) $\underline{C}_{D E}(X)=\left\{x \in U \mid \exists K \in K_{x}, K \subseteq X\right\}, \bar{C}_{D E}(X)=\left\{x \in U \mid \exists K \in K_{x}, K \cap X \neq \emptyset\right\}$.

(DA) $\underline{C}_{D A}(X)=\left\{x \in U \mid \forall K \in K_{x}, K \subseteq X\right\}, \bar{C}_{D A}(X)=\left\{x \in U \mid \forall K \in K_{x}, K \cap X \neq \emptyset\right\}$.

(GC) $\underline{C}_{G C}(X)=\{K \in C \mid K \subseteq X\}, \bar{C}_{G C}(X)=\{K \in C \mid K \cap X \neq \emptyset\}$.

Some necessary properties of DE, DA, and GC have been selected from [? ] and enumerated at Theorem 2.

Theorem 2. Let $(U, C)$ be a SMC approximation space. For any $X, Y \subseteq U$,

a) $\underline{C}_{D A}(X) \subseteq X \subseteq \bar{C}_{D A}(X)$ and $\underline{C}_{D E}(X) \subseteq X \subseteq \bar{C}_{D E}(X)$.

b) If $X \subseteq Y$, then $\underline{C}_{D A}(X) \subseteq \underline{C}_{D A}(Y), \bar{C}_{D A}(X) \subseteq \bar{C}_{D A}(Y), \underline{C}_{D E}(X) \subseteq \underline{C}_{D E}(Y), \bar{C}_{D E}(X) \subseteq \bar{C}_{D E}(Y)$.

c) $\underline{C}_{D A}(X \cap Y)=\underline{C}_{D A}(X) \cap \underline{C}_{D A}(Y), \bar{C}_{D A}(X \cup Y) \supseteq \bar{C}_{D A}(X) \cup \bar{C}_{D A}(Y)$. $\underline{\underline{C}}_{D E}(X \cap Y) \subseteq \underline{C}_{D E}(X) \cap \underline{\underline{C}}_{D E}(Y), \bar{C}_{D E}(X \cup Y)=\bar{C}_{D E}(X) \cup \bar{C}_{D E}(Y)$.

d) $\bar{C}_{D E}\left(\bar{C}_{D E}(X)\right) \supseteq \bar{C}_{D E}(X), \bar{C}_{D E}\left(\underline{C}_{D E}(X)\right) \supseteq \underline{C}_{D E}(X)$.

e) $\underline{C}_{D A}\left(\underline{C}_{D A}(X)\right) \subseteq \underline{C}_{D A}(X), \underline{C}_{D A}\left(\bar{C}_{D A}(X)\right) \subseteq \bar{C}_{D A}(X)$.

f) $\underline{C}_{D A}(X)=\left(\bar{C}_{D E}\left(X^{c}\right)\right)^{c}, \bar{C}_{D A}(X)=\left(\underline{C}_{D E}\left(X^{c}\right)\right)^{c}, \underline{C}_{D E}(X)=\left(\bar{C}_{D A}\left(X^{c}\right)\right)^{c}, \bar{C}_{D E}(X)=\left(\underline{C}_{D A}\left(X^{c}\right)\right)^{c}$.

g) $\underline{C}_{D E}(X)=\cup\{K \in C \mid K \subseteq X\}=\cup_{K \in \underline{C}_{G C}(X)} K, \bar{C}_{D E}(X)=\cup\{K \in C \mid K \cap X \neq \emptyset\}=\cup_{K \in \bar{C}_{G C}(X)} K$.

h) $\underline{C}_{D A}(X)=\cup_{K \in \underline{C}_{G C}(X)} K-\cup\left\{K_{1} \cap K_{2} \mid K_{1} \in \underline{C}_{G C}(X), K_{2} \notin \underline{C}_{G C}(X)\right\}$.

$\overline{\bar{C}}_{D A}(X)=\cup_{K \in \bar{C}_{G C}(X)} K-\cup\left\{K_{1} \cap K_{2} \mid K_{1} \in \bar{C}_{G C}(X), K_{2} \notin \overline{\bar{C}}_{G C}(X)\right\}$.

Inspired by the evolution of tolerance relations on set-valued information systems, enhancing the influence of single-valued records or limiting the interference from set-valued records can efficiently improve the approximation quality of approximation sets. Based on semi-monolayer covering approximation space, a new operator's form is designed to differentiate reliable elements (single-valued records) from controversial elements (set-valued records) in approximation operators. In the new approximation operators (DE0, DA0, and GC0), the reliable set of the blocks will take place of the block itself to participate in the computation, as shown in Definition 8.

Definition 8. Let $(U, C)$ be a SMC approximation space, and $X \subseteq U$, the reliable semi-monolayer covering approximation operators, namely DEO,DAO and GCO, are defined as follows:

(DEO) $\underline{C}_{D E 0}(X)=\left\{x \in U \mid \exists K \in K_{x}, K_{0} \subseteq X\right\}, \bar{C}_{D E 0}(X)=\left\{x \in U \mid \exists K \in K_{x}, K_{0} \cap X \neq \emptyset\right\}$.

(DAO) $\underline{C}_{D A 0}(X)=\left\{x \in U \mid \forall K \in K_{x}, K_{0} \subseteq X\right\}, \bar{C}_{D A 0}(X)=\left\{x \in U \mid \forall K \in K_{x}, K_{0} \cap X \neq \emptyset\right\}$.

(GCO) $\underline{C}_{G C 0}(X)=\left\{K \in C \mid K_{0} \subseteq X\right\}, \bar{C}_{G C 0}(X)=\left\{K \in C \mid K_{0} \cap X \neq \emptyset\right\}$.

Through comparison between Theorem 2, 3, 4 and 5, we can easily find the inheritance and improvement between DE, DA and DE0, DA0.

Theorem 3. Let $(U, C)$ be a SMC approximation space. For any $X, Y \subseteq U$,

a) $\bar{C}_{D E 0}(U)=\underline{C}_{D E 0}(U)=U, \bar{C}_{D E 0}(\emptyset)=\underline{C}_{D E 0}(\emptyset)=\emptyset$;

b) If $X \subseteq Y$, then $\underline{C}_{D E 0}(X) \subseteq \underline{C}_{D E 0}(Y), \bar{C}_{D E 0}(X) \subseteq \bar{C}_{D E 0}(Y)$;

c) $\underline{C}_{D E 0}(X \cap Y) \subseteq \underline{C}_{D E 0}(X) \cap \underline{C}_{D E 0}(Y), \bar{C}_{D E 0}(X \cup Y)=\bar{C}_{D E 0}(X) \cup \bar{C}_{D E 0}(Y)$;

d) $\underline{C}_{D E 0}\left(\underline{C}_{D E 0}(X)\right)=\bar{C}_{D E 0}\left(\underline{C}_{D E 0}(X)\right)=\underline{C}_{D E 0}(X), \underline{C}_{D E 0}\left(\bar{C}_{D E 0}(X)\right)=\bar{C}_{D E 0}\left(\bar{C}_{D E 0}(X)\right)=\bar{C}_{D E 0}(X)$.

Theorem 4. Let $(U, C)$ be a SMC approximation space. For any $X, Y \subseteq U$,

a) $\underline{C}_{D A 0}(U)=\bar{C}_{D A 0}(U)=U, \underline{C}_{D A 0}(\emptyset)=\bar{C}_{D A 0}(\emptyset)=\emptyset$;

b) If $X \subseteq Y$, then $\underline{C}_{D A 0}(X) \subseteq \underline{C}_{D A 0}(Y), \bar{C}_{D A 0}(X) \subseteq \bar{C}_{D A 0}(Y)$;

c) $\underline{C}_{D A 0}(X \cap Y)=\underline{C}_{D A 0}(X) \cap \underline{C}_{D A 0}(Y), \bar{C}_{D A 0}(X \cup Y) \supseteq \bar{C}_{D A 0}(X) \cup \bar{C}_{D A 0}(Y)$;

d) $\underline{C}_{D A 0}\left(\underline{C}_{D A 0}(X)\right)=\bar{C}_{D A 0}\left(\underline{C}_{D A 0}(X)\right)=\underline{C}_{D A 0}(X), \underline{C}_{D A 0}\left(\bar{C}_{D A 0}(X)\right)=\bar{C}_{D A 0}\left(\bar{C}_{D A 0}(X)\right)=\bar{C}_{D A 0}(X)$.

Theorem 5. Let $(U, C)$ be a SMC approximation space. For any $X \subseteq U$,

a) $\underline{C}_{D A 0}\left(X^{c}\right)=\left(\bar{C}_{D E 0}(X)\right)^{c}, \underline{C}_{D E 0}\left(X^{c}\right)=\left(\bar{C}_{D A 0}(X)\right)^{c}$; 
b) $\bar{C}_{D A 0}\left(X^{c}\right)=\left(\underline{C}_{D E 0}(X)\right)^{c}, \bar{C}_{D E 0}\left(X^{c}\right)=\left(\underline{C}_{D A 0}(X)\right)^{c}$;

The proofs of Theorem 3, Theorem 4 and Theorem 5 will be postponed after Theorem 14 in Section 5.1.

The properties of DE0 and DA0 are substantially similar to DE and DA ones in overall impression, but there have some differences between them in the practical details. Idempotence is one of the differences. DE0 and DA0 are idempotent (Theorem 3-d and Theorem 4-d),

$$
\begin{aligned}
& \underline{C}_{D E 0}\left(\underline{C}_{D E 0}(X)\right)=\bar{C}_{D E 0}\left(\underline{C}_{D E 0}(X)\right)=\underline{C}_{D E 0}(X), \underline{C}_{D E 0}\left(\bar{C}_{D E 0}(X)\right)=\bar{C}_{D E 0}\left(\bar{C}_{D E 0}(X)\right)=\bar{C}_{D E 0}(X) \\
& \underline{C}_{D A 0}\left(\underline{C}_{D A 0}(X)\right)=\bar{C}_{D A 0}\left(\underline{C}_{D A 0}(X)\right)=\underline{C}_{D A 0}(X), \underline{C}_{D A 0}\left(\bar{C}_{D A 0}(X)\right)=\bar{C}_{D A 0}\left(\bar{C}_{D A 0}(X)\right)=\bar{C}_{D A 0}(X)
\end{aligned}
$$

but DE and DA are not (Theorem 2 - $d$ and e).

$$
\begin{aligned}
& \bar{C}_{D E}\left(\bar{C}_{D E}(X)\right) \supseteq \bar{C}_{D E}(X), \bar{C}_{D E}\left(\underline{C}_{D E}(X)\right) \supseteq \underline{C}_{D E}(X), \\
& \underline{C}_{D A}\left(\underline{C}_{D A}(X)\right) \subseteq \underline{C}_{D A}(X), \underline{C}_{D A}\left(\bar{C}_{D A}(X)\right) \subseteq \bar{C}_{D A}(X) .
\end{aligned}
$$

\section{Improvement of Approximation Quality Brought about by New Operator's Form in Reliable SMC Approx- imation Operators}

An improved operator's form not only optimizes the properties of approximation operator but also increase the approximation quality of approximation sets. However, nothing is perfect. We will clarify the achievement and the payment from the improvement in theory.

\subsection{Positive Effect from New Operator's Form in Reliable SMC Approximation Operators}

The relationship between DE0, DA0, GC0 and DE, DA, GC (Theorem 6) revealed the changes brought about by new operator's form.

Theorem 6. Let $(U, C)$ be a SMC approximation space and $U_{0}^{c}=U-U_{0}$. For any $X \subseteq U$,

a) $\underline{C}_{D A 0}(X)=\underline{C}_{D A}\left(X \cup U_{0}^{c}\right), \underline{C}_{D E 0}(X)=\underline{C}_{D E}\left(X \cup U_{0}^{c}\right)$;

b) $\bar{C}_{D A 0}(X)=\overline{\bar{C}}_{D A}\left(X_{0}\right), \bar{C}_{D E 0}(X)=\bar{C}_{D E}\left(X_{0}\right)$.

c) $\underline{C}_{D A 0}(X) \supseteq \underline{C}_{D A}(X), \underline{C}_{D E 0}(X) \supseteq \underline{C}_{D E}(X)$;

d) $\overline{\bar{C}}_{D A 0}(X) \subseteq \overline{\bar{C}}_{D A}(X), \overline{\bar{C}}_{D E 0}(X) \subseteq \overline{\bar{C}}_{D E}(X)$;

e) $\underline{C}_{G C 0}(X) \supseteq \underline{C}_{G C}(X), \bar{C}_{G C 0}(X) \subseteq \bar{C}_{G C}(X)$.

Proof. a) To accomplish the proof, it should be proven that $K_{0} \subseteq X \Longleftrightarrow K \subseteq X \cup U_{0}^{c}$ at first.

“”. $K-K_{0} \subseteq U_{0}^{c}$ and $K=K_{0} \cup\left(K-K_{0}\right)$ are clear. Thus $K=K_{0} \cup\left(K-K_{0}\right) \subseteq X \cup U_{0}^{c}$.

" then $K_{0} \cup\left(K-K_{0}\right)-U_{0}^{c} \subseteq X \cup U_{0}^{c}-U_{0}^{c}$ and $K_{0} \subseteq X_{0} \subseteq X$.

Because $K_{0} \subseteq X \Longleftrightarrow K \subseteq X \cup U_{0}^{c}$, the following equations hold:

$$
\begin{aligned}
\underline{C}_{D A 0}(X) & =\left\{x \in U \mid \forall K \in K_{x}, K_{0} \subseteq X\right\} \\
& =\left\{x \in U \mid \forall K \in K_{x}, K \subseteq X \cup U_{0}^{c}\right\}=\underline{C}_{D A}\left(X \cup U_{0}^{c}\right) \\
\underline{C}_{D E 0}(X) & =\left\{x \in U \mid \exists K \in K_{x}, K_{0} \subseteq X\right\} \\
& =\left\{x \in U \mid \exists K \in K_{x}, K \subseteq X \cup U_{0}^{c}\right\}=\underline{C}_{D E}\left(X \cup U_{0}^{c}\right) .
\end{aligned}
$$

b) For every $K \in C, K_{0} \cap X=\left(K \cap U_{0}\right) \cap X=K \cap\left(U_{0} \cap X\right)=K \cap X_{0}$. Thus,

$$
\begin{aligned}
\bar{C}_{D A 0}(X) & =\left\{x \in U \mid \forall K \in K_{x}, K_{0} \cap X \neq \emptyset\right\} \\
& =\left\{x \in U \mid \forall K \in K_{x}, K \cap X_{0} \neq \emptyset\right\}=\bar{C}_{D A}\left(X_{0}\right) \\
\bar{C}_{D E 0}(X) & =\left\{x \in U \mid \exists K \in K_{x}, K_{0} \cap X \neq \emptyset\right\} \\
& =\left\{x \in U \mid \exists K \in K_{x}, K \cap X_{0} \neq \emptyset\right\}=\bar{C}_{D E}\left(X_{0}\right)
\end{aligned}
$$


By Theorem 2-b,

$$
\begin{gathered}
\underline{C}_{D A 0}(X)=\underline{C}_{D A}\left(X \cup U_{0}^{c}\right) \supseteq \underline{C}_{D A}(X), \\
\underline{C}_{D E 0}(X)=\underline{C}_{D E}\left(X \cup U_{0}^{c}\right) \supseteq \underline{C}_{D E}(X), \\
\bar{C}_{D A 0}(X)=\bar{C}_{D A}\left(X_{0}\right) \supseteq \bar{C}_{D A}(X), \\
\bar{C}_{D E 0}(X)=\bar{C}_{D E}\left(X_{0}\right) \supseteq \bar{C}_{D E}(X) .
\end{gathered}
$$

Thus, c) and d) hold.

The proof about GC0 (Theorem 6-e) will be postponed after Theorem 14 in section 4.1.

Based on Theorem 6, the lower (upper) DE0 and DA0 approximation sets are bigger (smaller) than the DE and DA ones. Naturally, the dominance relation has been maintained into their projections on $U_{0}$. That is to say that the lower (upper) DE0 and DA0 approximation sets contain more (less) reliable elements than the DE and DA ones (Corollary 7).

Corollary 7. Let $(U, C)$ be a SMC approximation space. For every $X \subseteq U$,

a) $\underline{C}_{D A 0}(X) \cap U_{0} \supseteq \underline{C}_{D A}(X) \cap U_{0}$;

b) $\underline{C}_{D E 0}(X) \cap U_{0} \supseteq \underline{C}_{D E}(X) \cap U_{0}$;

c) $\bar{C}_{D A 0}(X) \cap U_{0} \subseteq \bar{C}_{D A}(X) \cap U_{0}$;

d) $\bar{C}_{D E 0}(X) \cap U_{0} \subseteq \bar{C}_{D E}(X) \cap U_{0}$;

Example 2. Let $(U, C)$ be a SMC approximation space, where $U=\left\{x_{1}, x_{2}, \ldots, x_{17}\right\}$ and $C=\left\{K_{1}, K_{2}, K_{3}, K_{4}\right\} . K_{10}=$ $\{1,2,3\}, K_{20}=\{6,7,8\}, K_{30}=\{14,15\}$ and $K_{40}=\{17\}$ are the reliable sets of $K_{1}=\{1,2,3,4,5,9,10,11\}, K_{2}=$ $\{4,5,6,7,8,11,12,14\}, K_{3}=\{9,10,11,14,15,16\}$ and $K_{4}=\{11,12,13,16,17\}$ correspondingly.

The approximated set is $X . X=\{1,2,3,4,6,9,10\}=K_{10} \cup\{4\} \cup\{6\} \cup\{9,10\}$, where $\{4\} \in K_{1} \cap K_{2},\{6\} \in K_{20}$ and $\{9,10\} \subseteq K_{2} \cap K_{3}$. Which approximation sets are closer to the approximated set?

$$
\begin{gathered}
\underline{C}_{D A}(X)=\underline{C}_{D E}(X)=\emptyset, \bar{C}_{D A}(X)=K_{10} \cup K_{20} \cup K_{30} \cup\{4,5\} \cup\{9,10\}, \bar{C}_{D E}(X)=K_{1} \cup K_{2} \cup K_{3} . \\
\underline{C}_{D A 0}(X)=K_{10}, \underline{C}_{D E 0}(X)=K_{1}, \bar{C}_{D A 0}(X)=K_{10} \cup K_{20} \cup\{4,5\}, \bar{C}_{D E 0}(X)=K_{1} \cup K_{2},
\end{gathered}
$$

As shown in Figure 3, the DEO and DAO approximation sets are closer to $X$ than the DE and DA ones.

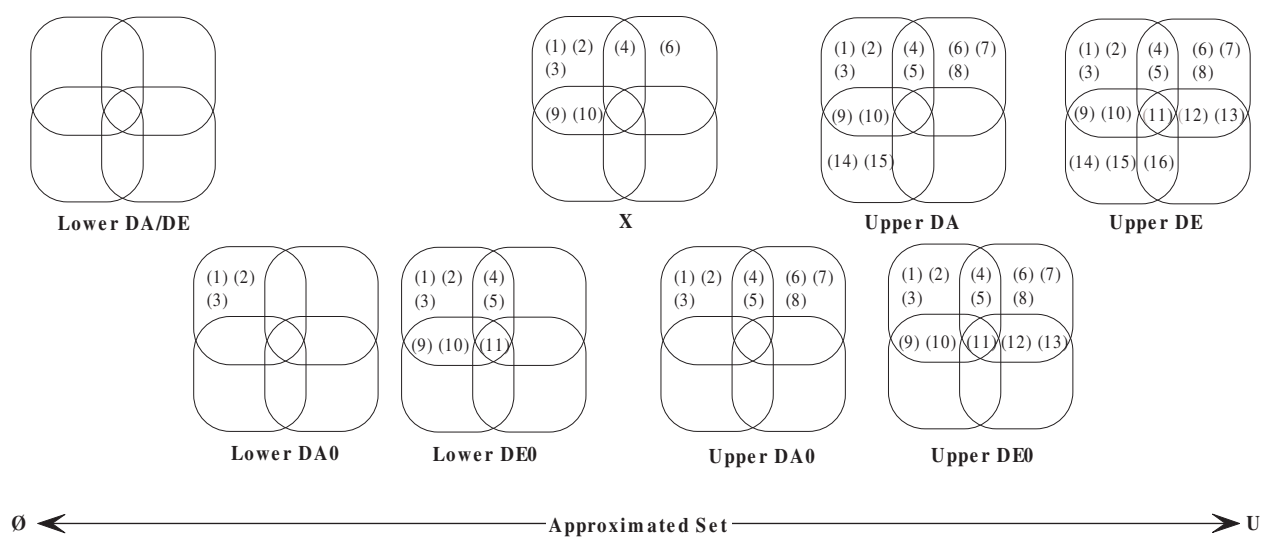

Figure 3: Approximation Sets of $X$ 


\subsection{Some Unaffected Approximated Sets}

Generally, the lower (upper) approximation sets of DE0 and DA0 are bigger (small) than the ones of DE and DA (Theorem 6). In this section, we will give some special approximated sets $X$ which DE0 and DA0 approximation sets are equal to the DE and DA ones respectively (Theorem 9). They are unaffected approximated sets for reliable semi-monolayer covering approximation operators. Theorem 9 can be consider as the complement of Theorem 6 .

Lemma 8. Let $C$ be $S M C$ on $U$ and $A \subseteq C$,

a) $X=\cup_{K_{i} \in A} K_{i}$. If $K_{0} \subseteq X$, then $K \subseteq X$.

b) $Y=\cup_{K_{i} \in A} K_{i}-\cup\left\{K_{1} \cap K_{2} \mid K_{1} \in A, K_{2} \notin A\right\}$. If $K \cap Y \neq \emptyset$, then $K_{0} \cap Y \neq \emptyset$.

Proof. a) Because the semi-monolayer covering is a special representative covering, SMC is exact, $K^{\prime} \in A \Longleftrightarrow K^{\prime} \subseteq$ $\cup_{K_{i} \in A} K_{i}=X$. If $K_{0} \subseteq X$, then there exists a block $K$ in $A$ of which $K_{0}$ is the reliable set. Otherwise, if $K \notin A$, the reliable element $x$ in $K_{0}$ can not be covered by the blocks in $A$. $x \notin X$. It is contradictory to the hypothesis $K_{0} \subseteq X$. Thus $K \subseteq X$.

b) If $K \cap Y \neq \emptyset$, then $K \in A$. Otherwise, if $K \notin A, K \cap \cup_{K^{\prime} \in A} K^{\prime} \subseteq \cup\left\{K_{1} \cap K_{2} \mid K_{1} \in A, K_{2} \notin A\right\}$. By the hypothesis of $Y, K \cap Y=\emptyset$. It is contradictory.

When $K \in A, K_{0} \subseteq K \subseteq \cup_{K_{i} \in A} K_{i}$. Because $K_{0}$ is a reliable set, $K_{0} \cap \cup\left\{K_{1} \cap K_{2} \mid K_{1} \in A, K_{2} \notin A\right\}=\emptyset$. Thus $K_{0} \subseteq \cup_{K_{i} \in A} K_{i}-\cup\left\{K_{1} \cap K_{2} \mid K_{1} \in A, K_{2} \notin A\right\}=Y$ and $K_{0} \cap Y \neq \emptyset$.

The sets satisfying the conditions in Lemma 8 are real and easy to find. They are precisely the SMC approximation sets, such as the approximation sets in Example 2.

$$
\begin{gathered}
\underline{C}_{D E 0}(X)=K_{1}, \bar{C}_{D E 0}(X)=K_{1} \cup K_{2}, \bar{C}_{D E}(X)=K_{1} \cup K_{2} \cup K_{3} . \\
\underline{C}_{D A 0}(X)=K_{1}-\left(\left(K_{1} \cap K_{2}\right) \cup\left(K_{1} \cap K_{3}\right) \cup\left(K_{1} \cap K_{4}\right)\right)=K_{1}-\cup\{\{4,5,11\},\{9,10,11\},\{11\}\}, \\
\bar{C}_{D A 0}(X)=K_{1} \cup K_{2}-\left(\left(K_{1} \cap K_{3}\right) \cup\left(K_{1} \cap K_{4}\right) \cup\left(K_{2} \cap K_{3}\right) \cup\left(K_{2} \cap K_{4}\right)\right)=K_{1} \cup K_{2}-\cup\{\{9,10,11\},\{11\},\{11,12,13\}\}, \\
\bar{C}_{D A}(X)=K_{1} \cup K_{2} \cup K_{3}-\left(\left(K_{1} \cap K_{4}\right) \cup\left(K_{2} \cap K_{4}\right) \cup\left(K_{3} \cap K_{4}\right)\right)=K_{1} \cup K_{2} \cup K_{3}-\cup\{\{11\},\{11,12,13\},\{11,16\}\} .
\end{gathered}
$$

$\underline{C}_{D E 0}(X), \bar{C}_{D E 0}(X)$ and $\bar{C}_{D E}(X)$ satisfy the condition in Lemma 8-a); $\underline{C}_{D A 0}(X), \bar{C}_{D A 0}(X)$ and $\bar{C}_{D A}(X)$ satisfy the condition in Lemma 8-b).

Theorem 9. Let $(U, C)$ be a SMC approximation space. For any $A \subseteq C$,

a) If $X=\cup_{K_{i} \in A} K_{i}$, then $\underline{C}_{D A 0}(X)=\underline{C}_{D A}(X), \underline{C}_{D E 0}(X)=\underline{C}_{D E}(X), \underline{C}_{G C 0}(X)=\underline{C}_{G C}(X)$.

b) If $Y=\cup_{K_{i} \in A} K_{i}-\cup\left\{K_{1} \cap K_{2} \mid K_{1} \in A, K_{2} \notin A\right\}$, then $\bar{C}_{D A 0}(Y)=\bar{C}_{D A}(Y), \bar{C}_{D E 0}(Y)=\bar{C}_{D E}(Y), \bar{C}_{G C 0}(Y)=\bar{C}_{G C}(Y)$.

Proof. By Theorem 6-c, $\mathrm{d}$ and e,

$$
\begin{aligned}
& \underline{C}_{D A 0}(X) \supseteq \underline{C}_{D A}(X), \underline{C}_{D E 0}(X) \supseteq \underline{C}_{D E}(X), \underline{C}_{G C 0}(X) \supseteq \underline{C}_{G C}(X), \\
& \bar{C}_{D A 0}(Y) \subseteq \bar{C}_{D A}(Y), \bar{C}_{D E 0}(Y) \subseteq \bar{C}_{D E}(Y), \bar{C}_{G C 0}(Y) \subseteq \bar{C}_{G C}(Y) .
\end{aligned}
$$

Thus we only need to prove that

$$
\begin{aligned}
& \underline{C}_{D A 0}(X) \subseteq \underline{C}_{D A}(X), \underline{C}_{D E 0}(X) \subseteq \underline{C}_{D E}(X), \underline{C}_{G C 0}(X) \subseteq \underline{C}_{G C}(X), \\
& \bar{C}_{D A 0}(Y) \supseteq \bar{C}_{D A}(Y), \bar{C}_{D E 0}(Y) \supseteq \bar{C}_{D E}(Y), \bar{C}_{G C 0}(Y) \supseteq \bar{C}_{G C}(Y) .
\end{aligned}
$$

a) By Lemma 8-a, if $X=\cup_{K_{i} \in A} K_{i}$ and $K_{0} \in X$, then $K \in X$. Therefore,

$$
\begin{aligned}
\underline{C}_{D A 0}(X) & =\left\{x \in U \mid \forall K \in K_{x}, K_{0} \subseteq X\right\} \\
& \subseteq\left\{x \in U \mid \forall K \in K_{x}, K \subseteq X\right\}=\underline{C}_{D A}(X) \\
\underline{C}_{D E 0}(X) & =\left\{x \in U \mid \exists K \in K_{x}, K_{0} \subseteq X\right\} \\
& \subseteq\left\{x \in U \mid \exists K \in K_{x}, K \subseteq X\right\}=\underline{C}_{D E}(X) \\
\underline{C}_{G C 0}(X) & =\left\{K \in C \mid K_{0} \subseteq X\right\} \\
& \subseteq\{K \in C \mid K \subseteq X\}=\underline{C}_{G C}(X) .
\end{aligned}
$$


b) By Lemma 8-b, if $Y=\cup_{K_{i} \in A} K_{i}-\cup\left\{K_{1} \cap K_{2} \mid K_{1} \in A, K_{2} \notin A\right\}$ and $K \cap Y \neq \emptyset$, then $K_{0} \cap Y \neq \emptyset$. Thus,

$$
\begin{aligned}
\bar{C}_{D A}(Y) & =\left\{x \in U \mid \forall K \in K_{x}, K \cap Y \neq \emptyset\right\} \\
& \subseteq\left\{x \in U \mid \forall K \in K_{x}, K_{0} \cap Y \neq \emptyset\right\}=\bar{C}_{D A 0}(X) \\
\bar{C}_{D E}(Y) & =\left\{x \in U \mid \exists K \in K_{x}, K \cap Y \neq \emptyset\right\} \\
& \subseteq\left\{x \in U \mid \exists K \in K_{x}, K_{0} \cap Y \neq \emptyset\right\}=\bar{C}_{D E 0}(Y) \\
\bar{C}_{G C}(Y) & =\{K \in C \mid K \cap Y \neq \emptyset\} \\
& \subseteq\left\{K \in C \mid K_{0} \cap Y \neq \emptyset\right\}=\bar{C}_{G C 0}(Y)
\end{aligned}
$$

4.3. Negative Effect from New Operator's Form in Reliable SMC Approximation Operators -- Weak Monotonicity

The new operator form makes it true that the approximation sets of DE0 and DA0 will be closer to the approximated sets than the ones of DE and DA in both $U$ and $U_{0}$. However, the improvement is not costless. The cost is the weak monotonicity between approximation sets and approximated set.

$$
\underline{C}_{D E 0}(X) \cap U_{0} \subseteq X_{0} \subseteq \bar{C}_{D E 0}(X) \cap U_{0}, \underline{C}_{D A 0}(X) \cap U_{0} \subseteq X_{0} \subseteq \bar{C}_{D A 0}(X) \cap U_{0},
$$

but

$$
\underline{C}_{D E 0}(X) \subseteq \bar{C}_{D E 0}(X), \underline{C}_{D A 0}(X) \subseteq \bar{C}_{D A 0}(X) .
$$

Theorem 10. Let $(U, C)$ be a SMC approximation space. For any $X \subseteq U$,

a) $\underline{C}_{D E 0}(X) \subseteq \bar{C}_{D E 0}(X)$.

b) $\underline{C}_{D A 0}(X) \subseteq \bar{C}_{D A 0}(X)$.

Proof. For every $K \in C$, if $K_{0} \subseteq X, K_{0} \cap X \neq \emptyset$. Thus the conclusions hold naturally.

DE0 and DA0 approximation sets can not keep the monotonicity with $X$. It is to say that the lower approximation sets of DE0 and DA0 maybe contain some elements out of $X$, and the upper approximation sets would miss some elements in $X$. Those phenomena have appeared in Example 2. The lower DE0 approximation set of $X$ overflowed the range of $X$ and contained some elements out of $X$.

$$
\underline{C}_{D E 0}(X)=X \cup\{5,11\}-\{6\} \nsubseteq X
$$

The upper DA0 approximation set missed some elements in $X$.

$$
\bar{C}_{D A 0}(X)=X \cup\{5,7,8\}-\{9,10\} \nsupseteq X .
$$

By observing, those outliers just are some controversial elements. If we remove controversial elements from approximation sets, the projects of DE0 and DA0 approximation sets and approximated set X on $U_{0}$ will satisfy the monotonicity as usual (Theorem 11).

Theorem 11. Let $(U, C)$ be a SMC approximation space. For any $X \subseteq U$,

a) $\underline{C}_{D E 0}(X) \cap U_{0} \subseteq X_{0} \subseteq \bar{C}_{D E 0}(X) \cap U_{0}$;

b) $\underline{C}_{D A 0}(X) \cap U_{0} \subseteq X_{0} \subseteq \bar{C}_{D A 0}(X) \cap U_{0}$.

Theorem 11 will be proven after Theorem 14 .

For the DE0 and DA0 approximation sets in Example 2, their projections on $U_{0}$ still satisfy the monotonicity.

$$
\underline{C}_{D E}(X) \cap U_{0} \subset \underline{C}_{D E 0}(X) \cap U_{0} \subset X_{0}=\{1,2,3,6\} \subset \bar{C}_{D E 0}(X) \cap U_{0} \subset \bar{C}_{D E}(X) \cap U_{0} .
$$

Theorem 6 and Corollary 7 show us that the lower (upper) approximation sets of DA0 and DE0 are bigger (smaller) than the ones of DE and DA correspondingly wherever in $U$ or $U_{0}$. It means that DE0 and DA0 own better approximation quality than DE and DA correspondingly. However, the improvement in DE0 and DA0 is not costless. To contain as much (little) reliable elements into lower (upper) DA0 and DE0 approximation sets as possible, some controversial elements have been involved in (rejected from) approximation sets by mistake. However, in consideration of the different importance of single-valued records and set-valued records in set-valued information systems, we believe that the improvement in DE0 and DA0 is worthwhile. 


\section{Efficient Computation of DE0 and DA0 Approximation Sets}

The efficient computation of approximation sets is another important topic for rough set theory. As is well-known that traversing the whole universe to construct the neighborhood for every object is a very expensive action, especially for the large-scale universe. If the calculations of approximation sets are executed at the information granule level, the speedup is self-explanatory. Of course, there is a prerequisite for this transformation. Whatever the granule-based form or the dot-based form of approximation operator must lead to the same approximation set.

\subsection{Granular Representation of DEO and DAO Approximation Sets Based on "Block" in SMC Level}

If $x$ is a reliable element, there exists only one block $K$ in $K_{x}$. For the description of the reliable element $K_{x}$, the implications of " $\forall K \in K_{x}$ " and " $\exists K \in K_{x}$ " in DE0 and DA0 are same. While a reliable element $x$ belongs to $\underline{C}_{D A 0}(X)$, the reliable element very naturally belongs to $\underline{C}_{D E 0}(X)$. In other words, the DE0 and DA0 approximation sets contain the same reliable elements.

Theorem 12. Let $(U, C)$ be a SMC approximation space. For any $X \subseteq U$,

a) $\underline{C}_{D A 0}(X) \cap U_{0}=\underline{C}_{D E 0}(X) \cap U_{0}=\underline{C}_{D A 0}\left(X \cap U_{0}\right) \cap U_{0}=\underline{C}_{D E 0}\left(X \cap U_{0}\right) \cap U_{0}$;

b) $\bar{C}_{D A 0}(X) \cap U_{0}=\bar{C}_{D E 0}(X) \cap U_{0}=\overline{\bar{C}}_{D A 0}\left(X \cap U_{0}\right) \cap U_{0}=\overline{\bar{C}}_{D E 0}\left(X \cap U_{0}\right) \cap U_{0}$;

Theorem 12 is the theoretical basis of piecewise constructing the DE0 and DA0 approximation sets, and it will be proven after Theorem 14.

The projections of the DA0 and DE0 approximation sets on $U_{0}$ are the same (Theorem 12). It means that the differences between the DA0 and DE0 approximation sets only are some controversial elements rather than the reliable elements. For example, the DA0 and DE0 approximation sets of $X$ in Example 2.

$$
\underline{C}_{D A 0}(X) \cap U_{0}=\underline{C}_{D E 0}(X) \cap U_{0}=K_{10}, \bar{C}_{D A 0}(X) \cap U_{0}=\bar{C}_{D E 0}(X) \cap U_{0}=K_{10} \cup K_{20} .
$$

Their projections of the DA0 and DE0 approximation sets on $U_{0}$ are the same, but the controversial elements of them are different.

$$
\begin{gathered}
\underline{C}_{D E 0}(X)=K_{10} \cup\{4,5,9,10,11\}, \bar{C}_{D E 0}(X)=K_{10} \cup K_{20} \cup\{4,5,9,10,11,12,13\} ; \\
\underline{C}_{D A 0}(X)=K_{10}, \bar{C}_{D A 0}(X)=K_{10} \cup K_{20} \cup\{4,5\} .
\end{gathered}
$$

DE0 and DA0 approximation operators inherit from DE and DA ones. The granular forms of DE0 and DA0 are also developed from the ones of DE and DA, which have been shown in [? ]. Lemma 13 is just the bridge between DE0 (DA0) and DE (DA).

Lemma 13. Let $C$ be $S M C$ on $U$ and $A \subseteq C$. For any $K \in C, X \subseteq U$.

a) $K \subseteq X \cup U_{0}^{c} \Longleftrightarrow K_{0} \subseteq X$;

b) $K \cap X_{0}=K_{0} \cap X$;

c) $\cup_{K_{i} \in A} K_{i} \cap K_{0} \neq \emptyset \Longleftrightarrow K \in A \Longleftrightarrow K_{0} \subseteq \cup_{K_{i} \in A} K_{i}$;

d) $K_{0} \cap\left(\cup_{K_{i} \in A} K_{i}-Y\right) \neq \emptyset \Longleftrightarrow K \in A \Longleftrightarrow K_{0} \subseteq\left(\cup_{K_{i} \in A} K_{i}-Y\right)$, where $Y=\cup\left\{K_{1} \cap K_{2} \mid K_{1} \in A, K_{2} \notin A\right\}$.

Proof. a) “ $\Longrightarrow$ " $K$ can be divided into $K_{0}$ and $K-K_{0} . U_{0}^{c}$ can be divided into $K-K_{0}$ and $U_{0}^{c}-\left(K-K_{0}\right)$ too.

$$
K_{0}=K-\left(K-K_{0}\right) \subseteq\left(X \cup U_{0}^{c}\right)-\left(K-K_{0}\right) \subseteq\left(X-\left(K-K_{0}\right)\right) \cup\left(U_{0}^{c}-\left(K-K_{0}\right)\right) \subseteq X \cup\left(U_{0}^{c}-\left(K-K_{0}\right)\right) .
$$

Because $U_{0}^{c} \cap U_{0}=\emptyset, U_{0}^{c} \cap K_{0}=\emptyset$ and then $\left(U_{0}^{c}-\left(K-K_{0}\right)\right) \cap K_{0}=\emptyset$. Thus $K_{0} \subseteq X$.

" $\Longleftarrow$ "If $K_{0} \subseteq X$, then $K=K_{0} \cup\left(K-K_{0}\right) \subseteq X \cup\left(K-K_{0}\right) \subseteq X \cup U_{0}^{c}$.

Thus $K \subseteq X \cup U_{0}^{c} \Longleftrightarrow K_{0} \subseteq X$.

b) It obviously holds that $K \cap X_{0}=K \cap X \cap U_{0}=K_{0} \cap X$.

c) Step 1: $K_{0} \cap \cup_{K_{i} \in A} K_{i} \neq \emptyset \Longrightarrow K \in A$.

If $K_{0} \cap \cup_{K_{i} \in A} K_{i} \neq \emptyset$, there exists $x \in K_{0} \cap \cup_{K_{i} \in A} K_{i}$. It means that $x \in K_{0}$ and $x \in \cup_{K_{i} \in A} K_{i}$. Furthermore, $x \in \cup_{K_{i} \in A} K_{i}$ means that there exists $K^{\prime} \in A$ satisfying that $x \in K^{\prime} . x \in K_{0}$ means $x$ is a reliable element of $K$. There exists only one block in $C$ containing $x$. Thus $K=K^{\prime}$ and $K \in A$. 
Step 2: $K \in A \Longrightarrow K_{0} \subseteq \cup_{K_{i} \in A} K_{i}$.

Because $C$ is exact, $K \in A$ means $K \subseteq \cup_{K_{i} \in A} K_{i}$ and then $K_{0} \subseteq \cup_{K_{i} \in A} K_{i}$.

Step 3: $K_{0} \subseteq \cup_{K_{i} \in A} K_{i} \Longrightarrow \cup_{K_{i} \in A} K_{i} \cap K_{0} \neq \emptyset$. It is clear.

Above all, c) holds.

d) For every $x \in Y, x$ is a controversial element. $x \notin U_{0}$ and $K_{0} \cap Y=\emptyset$.

Step 1: $K_{0} \cap\left(\cup_{K_{i} \in A} K_{i}-Y\right) \neq \emptyset \Longrightarrow K \in A$.

$K_{0} \cap\left(\cup_{K_{i} \in A} K_{i}-Y\right)=\left(\cup_{K_{i} \in A} K_{i} \cap K_{0}\right) \cap\left(K_{0} \cap Y^{c}\right)=\left(\cup_{K_{i} \in A} K_{i} \cap K_{0}\right) \cap K_{0}=\cup_{K_{i} \in A} K_{i} \cap K_{0}$. According to the conclusion of c), if $K_{0} \cap\left(\cup_{K_{i} \in A} K_{i}-Y\right) \neq \emptyset$, then $K \in A$.

Step 2: $K \in A \Longrightarrow K_{0} \subseteq \cup_{K_{i} \in A} K_{i}-Y$.

Because $K \in A \Leftrightarrow K_{0} \subseteq \cup_{K_{i} \in A} K_{i}$ and $K_{0} \cap Y=\emptyset, K_{0}=K_{0}-Y \subseteq \cup_{K_{i} \in A} K_{i}-Y$.

Step 3: $K_{0} \subseteq \cup_{K_{i} \in A} K_{i}-Y \Longrightarrow K_{0} \cap\left(\cup_{K_{i} \in A} K_{i}-Y\right) \neq \emptyset$.

$K_{0} \cap\left(\cup_{K_{i} \in A} K_{i}-Y\right) \neq \emptyset$ is a direct result of $K_{0} \subseteq \cup_{K_{i} \in A} K_{i}-Y$.

Above all, d) holds.

Theorem 14. Let $(U, C)$ be a SMC approximation space, $U_{0}^{c}=U-U_{0}$. For any $X \subseteq U$,

a) $\underline{C}_{D E 0}(X)=\cup\left\{K \in C \mid K \subseteq\left(X \cup U_{0}^{c}\right)\right\}=\cup\left\{K \in C \mid K_{0} \subseteq X\right\}=\cup_{K_{i} \in A} K_{i}$,

b) $\bar{C}_{D E 0}(X)=\cup\left\{K \in C \mid K \cap X_{0} \neq \emptyset\right\}=\cup\left\{K \in C \mid K_{0} \cap X \neq \emptyset\right\}=\cup_{K_{i} \in B} K_{i}$,

c) $\underline{C}_{D A 0}(X)=\cup_{K_{i} \in A} K_{i}-\cup\left\{K_{1} \cap K_{2} \mid K_{1} \in A, K_{2} \notin A\right\}$,

d) $\bar{C}_{D A 0}(X)=\cup_{K_{i} \in B} K_{i}-\cup\left\{K_{1} \cap K_{2} \mid K_{1} \in B, K_{2} \notin B\right\}$.

where $A=\underline{C}_{G C 0}(X)$ and $B=\bar{C}_{G C 0}(X)$.

Proof. By the relations between DE, DA and DE0, DA0 (Theorem 6-a and b) and the granule-based forms of DE and DA (Theorem 2-e and $\mathrm{f}$ ), the following equations hold that

$$
\begin{gathered}
\underline{C}_{D E 0}(X)=\underline{C}_{D E}\left(X \cup U_{0}^{C}\right)=\cup_{K \in A} K, \\
\bar{C}_{D E 0}(X)=\bar{C}_{D E}\left(X_{0}\right)=\cup_{K \in B} K, \\
\underline{C}_{D A 0}(X)=\underline{C}_{D A}\left(X \cup U_{0}^{C}\right)=\cup_{K \in A} K-\cup\left\{K_{1} \cap K_{2} \mid K_{1} \in A, K_{2} \notin A\right\}, \\
\bar{C}_{D A 0}(X)=\bar{C}_{D A}\left(X_{0}\right)=\cup_{K \in B} K-\cup\left\{K_{1} \cap K_{2} \mid K_{1} \in B, K_{2} \notin B\right\},
\end{gathered}
$$

where $A=\underline{C}_{G C}\left(X \cup U_{0}^{C}\right)$ and $B=\bar{C}_{G C}\left(X_{0}\right)$.

Based on Lemma 13-a and $\mathrm{b}$, we can prove $A$ and $B$ are another forms of the lower and upper GC0 approximation set of $X$.

$$
\begin{gathered}
A=\underline{C}_{G C}\left(X \cup U_{0}^{C}\right)=\left\{K \in C \mid K \subseteq X \cup U_{0}^{C}\right\}=\left\{K \in C \mid K_{0} \subseteq X\right\}=\underline{C}_{G C 0}(X), \\
B=\bar{C}_{G C}\left(X_{0}\right)=\left\{K \in C \mid K \cap X_{0} \neq \emptyset\right\}=\left\{K \in C \mid K_{0} \cap X \neq \emptyset\right\}=\bar{C}_{G C 0}(X) .
\end{gathered}
$$

The DE0 and DA0 approximation sets have the same reliable elements. The only differences exist in the controversial elements. Theorem 12 only declares the existence of the differences, but Theorem 14 provides a quantitative statement for the differences. Furthermore, Theorem 14 gives us a way to rebuild DE0 and DA0 approximation sets based on the GC0 approximation sets.

Based on Theorem 14, some properties of DE0 and DA0 become more intelligible and can be easily proved, such as Theorem 3, Theorem 4, Theorem 6-e, Theorem 11, and Theorem 12.

Proof of Theorem 3. By the definition of DE0, a) and b) are easy to prove.

c) By Theorem 2-c, Theorem 6-a and 6-b, it holds that

$$
\begin{aligned}
\underline{C}_{D E 0}(X \cap Y) & =\underline{C}_{D E}\left((X \cap Y) \cup U_{0}^{C}\right)=\underline{C}_{D E}\left(\left(X \cup U_{0}^{C}\right) \cap\left(Y \cup U_{0}^{C}\right)\right) \\
& \subseteq \underline{C}_{D E}\left(X \cup U_{0}^{C}\right) \cap \underline{C}_{D E}\left(Y \cup U_{0}^{C}\right)=\underline{C}_{D E 0}(X) \cap \underline{C}_{D E 0}(Y) \\
\bar{C}_{D E 0}(X \cup Y) & =\bar{C}_{D E}\left((X \cup Y) \cap U_{0}\right)=\bar{C}_{D E}\left(X_{0} \cup Y_{0}\right) \\
& =\bar{C}_{D E}\left(X_{0}\right) \cup \bar{C}_{D E}\left(Y_{0}\right)=\bar{C}_{D E 0}(X) \cup \bar{C}_{D E 0}(Y) .
\end{aligned}
$$


d) Let $A_{1}=\left\{K \in C \mid K_{0} \subseteq X\right\}$ and $A_{2}=\left\{K \in C \mid K_{0} \cap X \neq \emptyset\right\} . \underline{C}_{D E 0}(X)=\cup_{K_{i} \in A_{1}} K_{i}$ and $\bar{C}_{D E 0}(X)=\cup_{K_{i} \in A_{2}} K_{i}$ (Theorem 14-a and b). Additionally, by Lemma 13-c, $\cup_{K_{i} \in A_{1}} K_{i} \cap K_{0} \neq \bar{\emptyset} \Longleftrightarrow K \in A_{1}$ and $K_{0} \subseteq \cup_{K_{i} \in A_{2}} K_{i} \Longleftrightarrow K \in A_{2}$. Thus, the following equation holds.

$$
\begin{aligned}
\underline{C}_{D E 0}\left(\underline{C}_{D E 0}(X)\right) & =\left\{x \mid \exists K \in K_{x}, K \in \underline{C}_{D E 0}(X)\right\}=\left\{x \mid \exists K \in K_{x}, K \in \cup_{K_{i} \in A_{1}} K_{i}\right\} \\
& =\left\{x \mid \exists K \in K_{x}, K \in A_{1}\right\}=\left\{x \mid \exists K \in K_{x}, K_{0} \subseteq X\right\}=\underline{C}_{D E 0}(X) ; \\
\bar{C}_{D E 0}\left(\underline{C}_{D E 0}(X)\right) & =\left\{x \mid \exists K \in K_{x}, K \cap \underline{C}_{D E 0}(X) \neq \emptyset\right\}=\left\{x \mid \exists K \in K_{x}, \cup_{K_{i} \in A_{1}} K_{i} \cap K \neq \emptyset\right\} \\
& =\left\{x \mid \exists K \in K_{x}, K \in A_{1}\right\}=\left\{x \mid \exists K \in K_{x}, K_{0} \subseteq X\right\}=\underline{C}_{D E 0}(X) ; \\
\underline{C}_{D E 0}\left(\bar{C}_{D E 0}(X)\right) & =\left\{x \mid \exists K \in K_{x}, K \in \bar{C}_{D E 0}(X)\right\}=\left\{x \mid \exists K \in K_{x}, K \in \cup_{K_{i} \in A_{2}} K_{i}\right\} \\
& =\left\{x \mid \exists K \in K_{x}, K \in A_{2}\right\}=\left\{x \mid \exists K \in K_{x}, K_{0} \cap X \neq \emptyset\right\}=\bar{C}_{D E 0}(X) ; \\
\bar{C}_{D E 0}\left(\bar{C}_{D E 0}(X)\right) & =\left\{x \mid \exists K \in K_{x}, K \cap \bar{C}_{D E 0}(X) \neq \emptyset\right\}=\left\{x \mid \exists K \in K_{x}, \cup_{K_{i} \in A_{2}} K_{i} \cap K \neq \emptyset\right\} \\
& =\left\{x \mid \exists K \in K_{x}, K \in A_{2}\right\}=\left\{x \mid \exists K \in K_{x}, K_{0} \cap X \neq \emptyset\right\}=\bar{C}_{D E 0}(X) .
\end{aligned}
$$

Proof of Theorem 4. By the definition of DA0, a) and b) are easy to prove.

c) By Theorem 2-b and Theorem 6-a and 6-b, it holds that

$$
\begin{aligned}
\underline{C}_{D A 0}(X \cap Y) & =\underline{C}_{D A}\left((X \cap Y) \cup U_{0}^{C}\right)=\underline{C}_{D A}\left(\left(X \cup U_{0}^{C}\right) \cap\left(Y \cup U_{0}^{C}\right)\right) \\
& =\underline{C}_{D A}\left(X \cup U_{0}^{C}\right) \cap \underline{C}_{D A}\left(Y \cup U_{0}^{C}\right)=\underline{C}_{D A 0}(X) \cap \underline{C}_{D A 0}(Y) \\
\bar{C}_{D A 0}(X \cup Y) & =\bar{C}_{D A}\left((X \cup Y) \cap U_{0}\right)=\bar{C}_{D A}\left(X_{0} \cup Y_{0}\right) \\
& \supseteq \bar{C}_{D A}\left(X_{0}\right) \cup \bar{C}_{D A}\left(Y_{0}\right)=\bar{C}_{D A 0}(X) \cup \bar{C}_{D A 0}(Y) .
\end{aligned}
$$

d) Let $A_{1}=\left\{K \in C \mid K_{0} \subseteq X\right\}, A_{2}=\left\{K \in C \mid K_{0} \cap X \neq \emptyset\right\}, Y_{1}=\cup\left\{K_{1} \cap K_{2} \mid K_{1} \in A_{1}, K_{2} \notin A_{1}\right\}$ and $Y_{2}=\cup\left\{K_{1} \cap K_{2} \mid K_{1} \in\right.$ $\left.A_{2}, K_{2} \notin A_{2}\right\}$. By Theorem 14-c and d, $\underline{C}_{D A 0}(X)=\cup_{K_{i} \in A_{1}} K_{i}-Y_{1}, \bar{C}_{D A 0}(X)=\cup_{K_{i} \in A_{2}} K_{i}-Y_{2}$. Additionally, by Lemma 13-d, $K_{0} \cap\left(\cup_{K_{i} \in A_{1}} K_{i}-Y_{1}\right) \neq \emptyset \Longleftrightarrow K \in A_{1}$ and $K_{0} \subseteq\left(\cup_{K_{i} \in A_{2}} K_{i}-Y_{2}\right) \Longleftrightarrow K \in A_{2}$. Thus, the following equation holds.

$$
\begin{aligned}
\underline{C}_{D A 0}\left(\underline{C}_{D A 0}(X)\right) & =\left\{x \mid \forall K \in K_{x}, K \subseteq \underline{C}_{D A 0}(X)\right\}=\left\{x \mid \forall K \in K_{x}, K \subseteq\left(\cup_{K_{i} \in A_{1}} K_{i}-Y_{1}\right)\right\} \\
& =\left\{x \mid \forall K \in K_{x}, K \in A_{1}\right\}=\left\{x \mid \forall K \in K_{x}, K_{0} \subseteq X\right\}=\underline{C}_{D A 0}(X) ; \\
\bar{C}_{D A 0}\left(\underline{C}_{D A 0}(X)\right) & =\left\{x \mid \forall K \in K_{x}, K \cap \underline{C}_{D E 0}(X) \neq \emptyset\right\}=\left\{x \mid \forall K \in K_{x}, K \cap\left(\cup_{K_{i} \in A_{1}} K_{i}-Y_{1}\right) \neq \emptyset\right\} \\
& =\left\{x \mid \forall K \in K_{x}, K \in A_{1}\right\}=\left\{x \mid \forall K \in K_{x}, K_{0} \subseteq X\right\}=\underline{C}_{D A 0}(X) ; \\
\underline{C}_{D A 0}\left(\bar{C}_{D A 0}(X)\right) & =\left\{x \mid \forall K \in K_{x}, K \subseteq \bar{C}_{D A 0}(X)\right\}=\left\{x \mid \forall K \in K_{x}, K \subseteq\left(\cup_{K_{i} \in A_{2}} K_{i}-Y_{2}\right)\right\} \\
& =\left\{x \mid \forall K \in K_{x}, K \in A_{2}\right\}=\left\{x \mid \forall K \in K_{x}, K_{0} \cap X \neq \emptyset\right\}=\bar{C}_{D A 0}(X) ; \\
\bar{C}_{D A 0}\left(\bar{C}_{D A 0}(X)\right) & =\left\{x \mid \forall K \in K_{x}, K \cap \bar{C}_{D A 0}(X) \neq \emptyset\right\}=\left\{x \mid \forall K \in K_{x}, K \cap\left(\cup_{K_{i} \in A_{2}} K_{i}-Y_{2}\right) \neq \emptyset\right\} \\
& =\left\{x \mid \forall K \in K_{x}, K \in A_{2}\right\}=\left\{x \mid \forall K \in K_{x}, K_{0} \cap X \neq \emptyset\right\}=\bar{C}_{D A 0}(X) .
\end{aligned}
$$

Proof of Theorem 5. Let $U_{0}^{C}=U-U_{0}$. Recall some conclusions in Theorem 2 and Theorem 6.

$$
\begin{gathered}
\underline{C}_{D A}(X)=\left(\bar{C}_{D E}\left(X^{c}\right)\right)^{c}, \underline{C}_{D E}(X)=\left(\bar{C}_{D A}\left(X^{c}\right)\right)^{c} ; \\
\bar{C}_{D A}(X)=\left(\underline{C}_{D E}\left(X^{c}\right)\right)^{c}, \bar{C}_{D E}(X)=\left(\underline{C}_{D A}\left(X^{c}\right)\right)^{c} . \\
\underline{C}_{D A 0}(X)=\underline{C}_{D A}\left(X \cup U_{0}^{C}\right), \underline{C}_{D E 0}(X)=\underline{C}_{D E}\left(X \cup U_{0}^{C}\right) ; \\
\bar{C}_{D A 0}(X)=\bar{C}_{D A}\left(X_{0}\right), \bar{C}_{D E 0}(X)=\bar{C}_{D E}\left(X_{0}\right) .
\end{gathered}
$$

According to the above conclusions, the following equations hold.

$$
\begin{aligned}
\underline{C}_{D A 0}\left(X^{c}\right) & =\underline{C}_{D A}\left(X^{c} \cup U_{0}^{C}\right)=\underline{C}_{D A}\left(\left(X \cap U_{0}\right)^{c}\right) \\
& =\left(\bar{C}_{D E}\left(X_{0}\right)\right)^{c}=\left(\bar{C}_{D E 0}(X)\right)^{c} ;
\end{aligned}
$$




$$
\begin{aligned}
\underline{C}_{D E 0}\left(X^{c}\right) & =\underline{C}_{D E}\left(X^{c} \cup U_{0}^{C}\right)=\underline{C}_{D E}\left(\left(X \cap U_{0}\right)^{c}\right) \\
& =\left(\bar{C}_{D A}\left(X_{0}\right)\right)^{c}=\left(\bar{C}_{D A 0}(X)\right)^{c} ; \\
\bar{C}_{D A 0}\left(X^{c}\right) & =\bar{C}_{D A}\left(\left(X^{c}\right) \cap U_{0}\right)=\bar{C}_{D A}\left(\left(X \cup U_{0}^{C}\right)^{c}\right) \\
& =\left(\underline{C}_{D E}\left(X \cup U_{0}^{C}\right)\right)^{c}=\left(\underline{C}_{D E 0}(X)\right)^{c} ; \\
\bar{C}_{D E 0}\left(X^{c}\right) & =\bar{C}_{D E}\left(\left(X^{c}\right) \cap U_{0}\right)=\bar{C}_{D E}\left(\left(X \cup U_{0}^{C}\right)^{c}\right) \\
& =\left(\underline{C}_{D A}\left(X \cup U_{0}^{C}\right)\right)^{c}=\left(\underline{C}_{D A 0}(X)\right)^{c} .
\end{aligned}
$$

Proof of Theorem 6-e. For any $X \subseteq U, \underline{C}_{D E 0}(X) \supseteq \underline{C}_{D E}(X)$. Because of Theorem 2-f and Theorem 14, $\underline{C}_{D E 0}(X)=$ $\cup_{K \in \underline{C}_{G C 0}(X)} K$ and $\underline{C}_{D E}(X)=\cup_{K \in \underline{C}_{G C}(X)} K$. Additionally $C$ is exact, thus $\underline{C}_{G C 0}(X) \supseteq \underline{C}_{G C}(X)$.

Similarly, $\bar{C}_{D E 0}(X)=\cup_{K \in \bar{C}_{G C O}(X)} K, \bar{C}_{D E}(X)=\cup_{K \in \bar{C}_{G C}(X)} K$ and $\bar{C}_{D E 0}(X) \subseteq \bar{C}_{D E}(X)$. Thus it holds that $\bar{C}_{G C 0}(X) \subseteq$ $\bar{C}_{G C}(X)$.

Proof of Theorem 11. a) According to Theorem 14, $\underline{C}_{D E 0}(X)=\cup\left\{K \in C \mid K_{0} \subseteq X\right\}$ and $\bar{C}_{D E 0}(X)=\cup\left\{K \in C \mid K_{0} \cap X \neq\right.$ $\emptyset\}$. Additionally, $K_{0} \subseteq X \Longleftrightarrow K_{0} \subseteq X_{0}$ and $K_{0} \cap X \neq \emptyset \Longleftrightarrow K_{0} \cap X_{0} \neq \emptyset$ are clear. Thus the following inequations hold.

$$
\begin{aligned}
\underline{C}_{D E 0}(X) \cap U_{0} & =\cup\left\{K \in C \mid K_{0} \subseteq X\right\} \cap U_{0}=\cup\left\{K_{0} \mid K \in C, K_{0} \subseteq X\right\} \\
& =\cup\left\{K_{0} \mid K \in C, K_{0} \subseteq X_{0}\right\} \subseteq X_{0} \\
\bar{C}_{D E 0}(X) \cap U_{0} & =\cup\left\{K \in C \mid K_{0} \cap X \neq \emptyset\right\} \cap U_{0}=\cup\left\{K_{0} \mid K \in C, K_{0} \cap X \neq \emptyset\right\} \\
& =\cup\left\{K_{0} \mid K \in C, K_{0} \cap X_{0} \neq \emptyset\right\} \supseteq X_{0} .
\end{aligned}
$$

Above all, $\underline{C}_{D E 0}(X) \cap U_{0} \subseteq X_{0} \subseteq \bar{C}_{D E 0}(X) \cap U_{0}$.

b) By Theorem 12- a and b, $\underline{C}_{D A 0}(X) \cap U_{0}=\underline{C}_{D E 0}(X) \cap U_{0}$ and $\bar{C}_{D A 0}(X) \cap U_{0}=\bar{C}_{D E 0}(X) \cap U_{0}$. It holds that

$$
\underline{C}_{D A 0}(X) \cap U_{0}=\underline{C}_{D E 0}(X) \cap U_{0} \subseteq X_{0} \subseteq \bar{C}_{D E 0}(X) \cap U_{0}=\bar{C}_{D A 0}(X) \cap U_{0}
$$

Proof of Theorem 12. For any $A \subseteq C$, Let $X_{1}=\cup_{K_{i} \in A} K_{i}, X_{2}=X_{1}-Y$ and $Y=\cup\left\{K_{1} \cap K_{2} \mid K_{1} \in A, K_{2} \notin A\right\}$. Because $x \in Y$ is a controversial element rather than a reliable element, $x \notin U_{0}$ and $Y \cap U_{0}=\emptyset$. It is logical that $Y^{c} \supseteq U_{0}$ and $Y^{c} \cap U_{0}=U_{0}$.

According to Theorem 14, if $A=\left\{K \mid K_{0} \subseteq X\right\}$, then $X_{1}=\underline{C}_{D E 0}(X)$ and $X_{2}=\underline{C}_{D A 0}(X)$. Thus

$$
\underline{C}_{D A 0}(X) \cap U_{0}=\left(\cup_{K_{i} \in A} K_{i}-Y\right) \cap U_{0}=\cup_{K_{i} \in A} K_{i} \cap Y^{c} \cap U_{0}=\cup_{K_{i} \in A} K_{i} \cap U_{0}=\underline{C}_{D E 0}(X) \cap U_{0}
$$

Furthermore, because $K_{0}$ is a reliable set, $K_{0} \cap X \neq \emptyset \Leftrightarrow K_{0} \cap X_{0} \neq \emptyset$ and $K_{0} \subseteq X \Leftrightarrow K_{0} \subseteq X_{0}$. By the definition of DE0 and DA0, $X \cap U_{0}$ and $X$ have same DE0/DA0 approximation sets. Therefore, the equations in this theorem hold.

$$
\begin{aligned}
& \underline{C}_{D E 0}\left(X \cap U_{0}\right) \cap U_{0}=\underline{C}_{D E 0}(X) \cap U_{0}=\underline{C}_{D A 0}\left(X \cap U_{0}\right) \cap U_{0}=\underline{C}_{D A 0}(X) \cap U_{0}, \\
& \bar{C}_{D E 0}\left(X \cap U_{0}\right) \cap U_{0}=\bar{C}_{D E 0}(X) \cap U_{0}=\bar{C}_{D A 0}\left(X \cap U_{0}\right) \cap U_{0}=\bar{C}_{D A 0}(X) \cap U_{0} .
\end{aligned}
$$

So far, we have proven all unproven conclusions.

\subsection{Improved Granular Representation of DEO and DAO Approximation Sets Based on "Cell" in Set-Valued Infor- mation System Level}

In the view of granular computing, the "block" in SMC is coarser than the "cell" in SVIS. The granular computation at SMC level seems to be faster. However, once integrating the construction of semi-monolayer covering in set-valued information systems, there is still much room for improvement in simplifying the calculations. Specifically, the process of calculating approximation sets can be divided into three steps.

- (Step 1) construct the blocks in semi-monolayer covering from SVIS or CSVIS; 
- (Step 2) calculate GC0 approximation sets;

- (Step 3) rebuild DA0 and DE0 approximation sets based on GC0 ones and the blocks in SMC

The controversial elements (set-valued records) were repeatedly computed twice in Step 1 and Step 3 respectively. Merging the repetitive steps can efficiently improve the computing speed. Therefore, an improved granular approach based on "Cell" is proposed to construct the DA0 and DE0 approximation sets directly from CSVIS rather than SMC.

Definition 9. Let $S=(U, A, V, f)$ be CSVIS. Cell $x=\{y \in U \vec{x}=\vec{y}\}$ is a cell on $S$. If the record in Cell is single-valued, the cell is called reliable cell, and the set of the reliable cell on $S$ is denoted RC. If the record in Cell is set-valued, the cell is called controversial cell, and the set of controversial cell is denoted CC. If for every $a \in A, x \in$ Cell $_{i}, y \in \operatorname{Cell}_{j} f(x, a) \subseteq f(y, a)$, we denoted Cell c Cell $_{y}$. For a controversial cell Cell,$R S(C e l l)=$ $\left\{\mathrm{Cell}_{r} \in \mathrm{RC} \mid \mathrm{Ce} \mathrm{ll}_{r} \Subset \mathrm{Cell}_{c}\right\}$ is the related reliable-cell set of Cell ${ }_{c}$.

Theorem 15 give an approach how to construct a block in SMC based on the cells on CSVIS.

Theorem 15. Let $(U, A, V, f)$ be CSVIS. If $K=\left\{y \mid \forall a \in A, f(x, a) \subseteq f(y, a), x \in\right.$ Cell $\left._{r}, C_{e l l} \in R C\right\}, C=\left\{K_{1}, K_{2}, \ldots, K_{n}\right\}$ is SMC on U. Let Cell,$\in R C$, there must exist a block $K_{r}$ satisfying that $K_{r 0}=$ Cell $_{r}$, and $K_{r}-$ Cell $_{r}=\cup\left\{C e l l_{c} \in\right.$ $\left.\mathrm{CC} \mid \mathrm{Cell} r \Subset \mathrm{Cell}_{c}\right\}$.

Proof. If $x \in$ Cell $_{r}$ and $C e l l_{r} \in R C, x$ is a single-valued record, $C=\left\{K_{1}, K_{2}, \ldots, K_{n}\right\}$ is SMC on $U$ by Theorem 1 .

The record $x \in C e l l_{r}$ and $C e l l_{r} \in R C$ in reliable cell is a single-valued one. $x$ only could belong to one block.

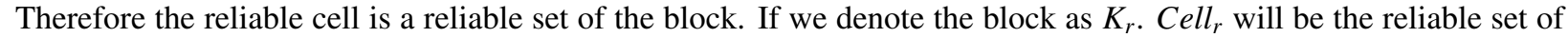
$K_{r}$.

If $y$ is a set-valued record and $y \in C e l l_{c}$, Cell $_{c} \in C C$, there exists $a_{0} \in A, f\left(x, a_{0}\right) \subset f\left(y, a_{0}\right), x \in$ Cell $_{r}$. Because Cell $_{r} \Subset$ Cell $_{c}$, Cell $_{c} \subset K_{r}$ by Definition 9, Furthermore, $y$ is a set-valued record, Cell $\cap \cap$ Cell $_{r}=\emptyset$. Therefore $\operatorname{Cell}_{c} \subseteq K_{r}-C_{e l l}$. If $y$ is a controversial element, there exists $a_{0} \in A, f\left(x, a_{0}\right) \subset f\left(y, a_{0}\right), x \in C_{\text {Cell }}$, Otherwise $y$ belong to $\mathrm{Cell}_{r}$ and $y$ is a reliable element. It is a contradictory. $\mathrm{Cell}_{c} \subseteq K_{r}-\mathrm{Cell}_{r}$. Therefore $K_{r}-\mathrm{Cell}_{r}=\cup\left\{\mathrm{Cell}_{c} \in\right.$ $C C \mid C e l l_{r} \Subset$ Cell $\left._{c}\right\}$.

By substituting the blocks in Theorem 14 with the combination of information cells in Theorem 15, we can calculate DE0 and DA0 approximation sets directly from CSVIS (complete set-valued information system). It avoids the repeated calculation of the controversial cells in essence.

Theorem 16. Let $(U, A, V, f)$ be CSVIS. RC and CC contain all reliable and controversial cells on CS VIS respectively. For any $X \subseteq U$, the DEO and DAO approximation sets of $X$ on CSVIS are as follows:

$$
\begin{gathered}
\underline{C}_{D E 0}(X)=\left(\cup\left\{\text { Cell }_{r} \in R C \mid \text { Cell }_{r} \in A^{\prime}\right\}\right) \cup\left(\cup\left\{\text { Cell }_{c} \in C C \mid R S\left(\text { Cell }_{c}\right) \cap A^{\prime} \neq \emptyset\right\}\right) ; \\
\bar{C}_{D E 0}(X)=\left(\cup\left\{\text { Cell }_{r} \in R C \mid \text { Cell }_{r} \in B^{\prime}\right\}\right) \cup\left(\cup\left\{\text { Cell }_{c} \in C C \mid R S\left(\text { Cell }_{c}\right) \cap B^{\prime} \neq \emptyset\right\}\right) ; \\
\underline{C}_{D A 0}(X)=\left(\cup\left\{\text { Cell }_{r} \in R C \mid \text { Cell }_{r} \in A^{\prime}\right\}\right) \cup\left(\cup\left\{\text { Cell }_{c} \in C C \mid R S\left(\text { Cell }_{c}\right) \subseteq A^{\prime}\right\}\right) ; \\
\bar{C}_{D A 0}(X)=\left(\cup\left\{\text { Cell }_{r} \in R C \mid C e l l_{r} \in B^{\prime}\right\}\right) \cup\left(\cup\left\{\text { Cell }_{c} \in C C \mid R S\left(\text { Cell }_{c}\right) \subseteq B^{\prime}\right\}\right) .
\end{gathered}
$$

where $A^{\prime}=\left\{\right.$ Cell $\left._{r} \in R C \mid C e l l_{r} \subseteq X\right\}$ and $B^{\prime}=\left\{\right.$ Cell $_{r} \in R C \mid$ Cell $\left._{r} \cap X \neq \emptyset\right\}$.

Proof. Let $A=\underline{C}_{G C 0}(X)$ and $B=\bar{C}_{G C 0}(X)$. By Theorem 15, Cell $r$ is the reliable set of $K_{r}$. Cell $r$ and $K_{r}$ are corresponding one by one. Therefore, for any $C_{e l l}$ in $A^{\prime}$ (or $B^{\prime}$ ), $K_{r}$ must be in $A$ (or $B$ ), vice versa.

For any $X \in U, \underline{C}_{D E 0}(X)=\cup\left\{K_{r} \mid K_{r} \in A\right\}, A=\underline{C}_{G C 0}(X)$ (Theorem 14). All of reliable sets in $\underline{C}_{D E 0}(X)$ are $\left\{C e l l_{r} \mid K_{r} \in A\right\}=\left\{C e l l_{r} \mid\right.$ Cell $\left._{r} \in A^{\prime}\right\}$. All of controversial elements in $\underline{C}_{D E 0}(X)$ are $\cup\left\{\right.$ Cell $_{c} \in$ CC Cell $_{r} \Subset$ Cell $_{c}$, Cell $_{r} \in$ $\left.A^{\prime}\right\}$ (by Theorem 15). Because of $\mathrm{Cell}_{r} \Subset \mathrm{Cell}_{c}, \mathrm{Cell}_{r} \in R S\left(\mathrm{Cell}_{c}\right)$. Therefore all of controversial elements in $\underline{C}_{D E 0}(X)$ is $\cup\left\{\right.$ Cell $_{c} \in C C \mid R S\left(\right.$ Cell $\left.\left._{c}\right) \cap A^{\prime} \neq \emptyset\right\}$, and then $\underline{C}_{D E 0}(X)=\left(\cup\left\{\right.\right.$ Cell $_{r} \in R C \mid$ Cell $\left.\left._{r} \in A^{\prime}\right\}\right) \cup\left(\cup\left\{\operatorname{Cell}_{c} \in C C \mid R S\left(C e l l_{c}\right) \cap A^{\prime} \neq\right.\right.$ $\emptyset\})$. Similarly, we can prove $\bar{C}_{D E 0}(X)=\left(\cup\left\{\right.\right.$ Cell $_{r} \in R C \mid$ Cell $\left.\left._{r} \in B^{\prime}\right\}\right) \cup\left(\cup\left\{\right.\right.$ Cell $_{c} \in C C \mid R S\left(\right.$ Cell $\left.\left.\left._{c}\right) \cap B^{\prime} \neq \emptyset\right\}\right)$ based on $\bar{C}_{D E 0}(X)=\cup\left\{K_{i} \mid K_{i} \in B\right\}$.

By Theorem 12, $\underline{C}_{D A 0}(X) \cap U_{0}=\underline{C}_{D E 0}(X) \cap U_{0}$ and $\bar{C}_{D A 0}(X) \cap U_{0}=\bar{C}_{D E 0}(X) \cap U_{0}$. DE0 and DA0 approximation sets have same reliable elements. Therefore $\cup\left\{\right.$ Cell $\left._{r} \in R C \mid C_{e l l} \in A^{\prime}\right\}$ contains all reliable element in $\underline{C}_{D A 0}(X)$. If 
Cell $_{c} \subseteq K_{1} \cap K_{2}$, Cell $_{1}$ and Cell $_{2}$ must be in $R S\left(C_{\text {ell }}\right.$ ) (by Theorem 15). By Theorem 14, $\underline{C}_{D A 0}(X)=\underline{C}_{D E 0}(X)-\cup\left\{K_{1} \cap\right.$ $\left.K_{2} \mid K_{1} \in A, K_{2} \notin A\right\}$. The controversial elements in $\underline{C}_{D A 0}(X)$ are $\left(\cup\left\{\right.\right.$ Cell $_{c} \in C C \mid R S\left(\right.$ Cell $\left.\left.\left._{c}\right) \cap A^{\prime} \neq \emptyset\right\}\right)-\left(\cup\left\{C e l l_{c} \in\right.\right.$ $\left.\left.C C \mid \exists C e l l_{j} \in R C, C_{e l l} \notin A^{\prime}, C_{e l l} \in R S\left(C_{j} e l l_{c}\right)\right\}\right)$. It also means that $R S\left(C_{e} l l_{c}\right)$ must be the subset of $A^{\prime}$. Therefore, $\underline{C}_{D A 0}(X)=\left(\cup\left\{\right.\right.$ Cell $_{r} \in R C \mid$ Cell $\left.\left._{r} \in A^{\prime}\right\}\right) \cup\left(\cup\left\{\right.\right.$ Cell $_{c} \in C C \mid R S\left(\right.$ Cell $\left.\left.\left._{c}\right) \subseteq A^{\prime}\right\}\right)$. Similarly, $\bar{C}_{D A 0}(X)=\left(\cup\left\{\right.\right.$ Cell $_{r} \in R C \mid C e l l_{r} \in$ $\left.\left.B^{\prime}\right\}\right) \cup\left(\cup\left\{C e l l_{c} \in C C \mid R S\left(C e l l_{c}\right) \subseteq B^{\prime}\right\}\right)$ based on $\bar{C}_{D A 0}(X)=\bar{C}_{D E 0}(X)-\cup\left\{K_{1} \cap K_{2} \mid K_{1} \in B, K_{2} \notin B\right\}$.

Example 3. Resume Example 2. Now, we can skip the construction of SMC and calculate the DEO and DAO approximation sets directly from information cells by Theorem 16.

The cells in CSVIS (Table 1) are Cell $1=\{1,2,3\}$, Cell $_{4}=\{4,5\}$, Cell $_{6}=\{6,7,8\}$, Cell $_{9}=\{9,10\}$, Cell $_{11}=\{11\}$, Cell $_{12}=\{12,13\}$, Cell $_{14}=\{14,15\}$, Cell $_{16}=\{16\}$ and Cell $_{17}=\{17\}$.

$$
\begin{gathered}
R C=\left\{\text { Cell }_{1}, \text { Cell }_{6}, \text { Cell }_{14}, \text { Cell }_{17}\right\}, \\
C C=\left\{\text { Cell }_{4}, \text { Cell }_{9}, \text { Cell }_{11}, \text { Cell }_{12}\right\} . \\
R S\left(\text { Cell }_{4}\right)=\left\{\text { Cell }_{1}, \text { Cell }_{6}\right\}, R S\left(\text { Cell }_{9}\right)=\left\{\text { Cell }_{1}, \text { Cell }_{14}\right\}, \\
R S\left(\text { Cell }_{11}\right)=\left\{\text { Cell }_{1}, \text { Cell }_{6}, \text { Cell }_{14}, \text { Cell }_{17}\right\}, \\
R S\left(\text { Cell }_{12}\right)=\left\{\text { Cell }_{6}, \text { Cell }_{17}\right\}, R S\left(\text { Cell }_{16}\right)=\left\{\text { Cell }_{14}, \text { Cell }_{17}\right\} .
\end{gathered}
$$

By referring to the relationship between $X=\{1,2,3,4,6,9,10\}$ and $R C$, we can get $A^{\prime}=\left\{\right.$ Cell $l_{1}$ and $B^{\prime}=$ $\left\{\mathrm{Cell}_{1}, \mathrm{Cell}_{6}\right\}$.

The final approximation set can be made from the reliable cells in $A^{\prime}$ and $B^{\prime}$ and the relative controversial cells by the rules on $D E O$ and $D A O$.

$$
\begin{gathered}
\underline{C}_{D A 0}(X)=\operatorname{Cell}_{1}=\{1,2,3\} ; \\
\bar{C}_{D A 0}(X)=\left(\text { Cell }_{1} \cup \text { Cell }_{6}\right) \cup \text { Cell }_{4}=\{1,2, \ldots, 8\} \\
\underline{C}_{D E 0}(X)=\operatorname{Cell}_{1} \cup\left(\text { Cell }_{4} \cup \text { Cell }_{9} \cup \operatorname{Cell}_{11}\right)=\{1,2,3,4,5,9,10,11\} \\
\bar{C}_{D E 0}(X)=\left(\text { Cell }_{1} \cup \text { Cell }_{6}\right) \cup\left(\text { Cell }_{4} \cup \text { Cell }_{9} \cup \text { Cell }_{11} \cup \text { Cell }_{12}\right)=\{1,2, \ldots, 13\} .
\end{gathered}
$$

\subsection{Efficient Computation of DEO and DAO Approximation Sets -- Granular Algorithms}

In CSVIS, the DE0 and DA0 approximation sets can be constructed from the reliable and controversial cells directly, as shown at Theorem 16. Following the idea in Theorem 16 and the steps in Example 3, we design Algorithm GAS to realize the granular computing of DE0 and DA0 approximation sets in CSVIS. The internal relations between those sub algorithms (Algorithm GAS-Init, GAS-RS, GAS-AB, and GAS-Appr) are shown in Figure 4.

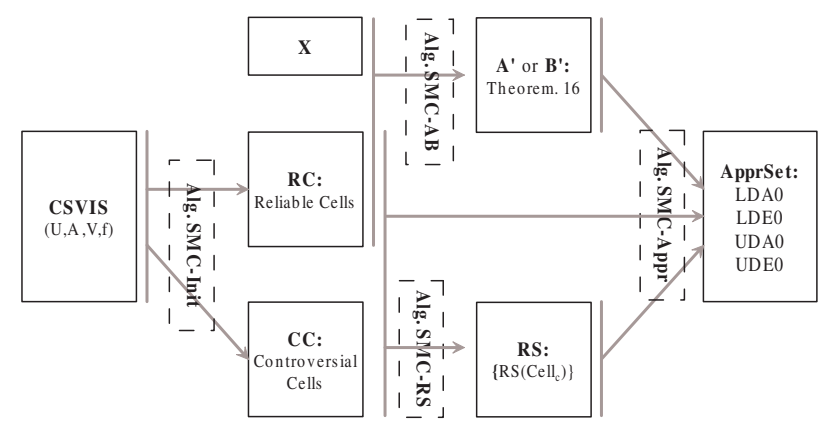

Figure 4: Relations between Algorithms

In Algorithm GAS-Init (Algorithm 1), the operation of traversing the whole universe has been performed only once, and the single-valued or set-valued records are distributed into $C C$ or $R C$ correspondingly. The time complexity is $O(|U|)$. In Algorithm GAS-RS (Algorithm 2), we will calculate the related reliable cell set $R S\left(C e l l_{c}\right) \in R S$ for every controversial cell $C e l l_{c} \in C C$. The time complexity of Algorithm SMC-RS is $O(|C C| \times|R C|)$. At the same time, 

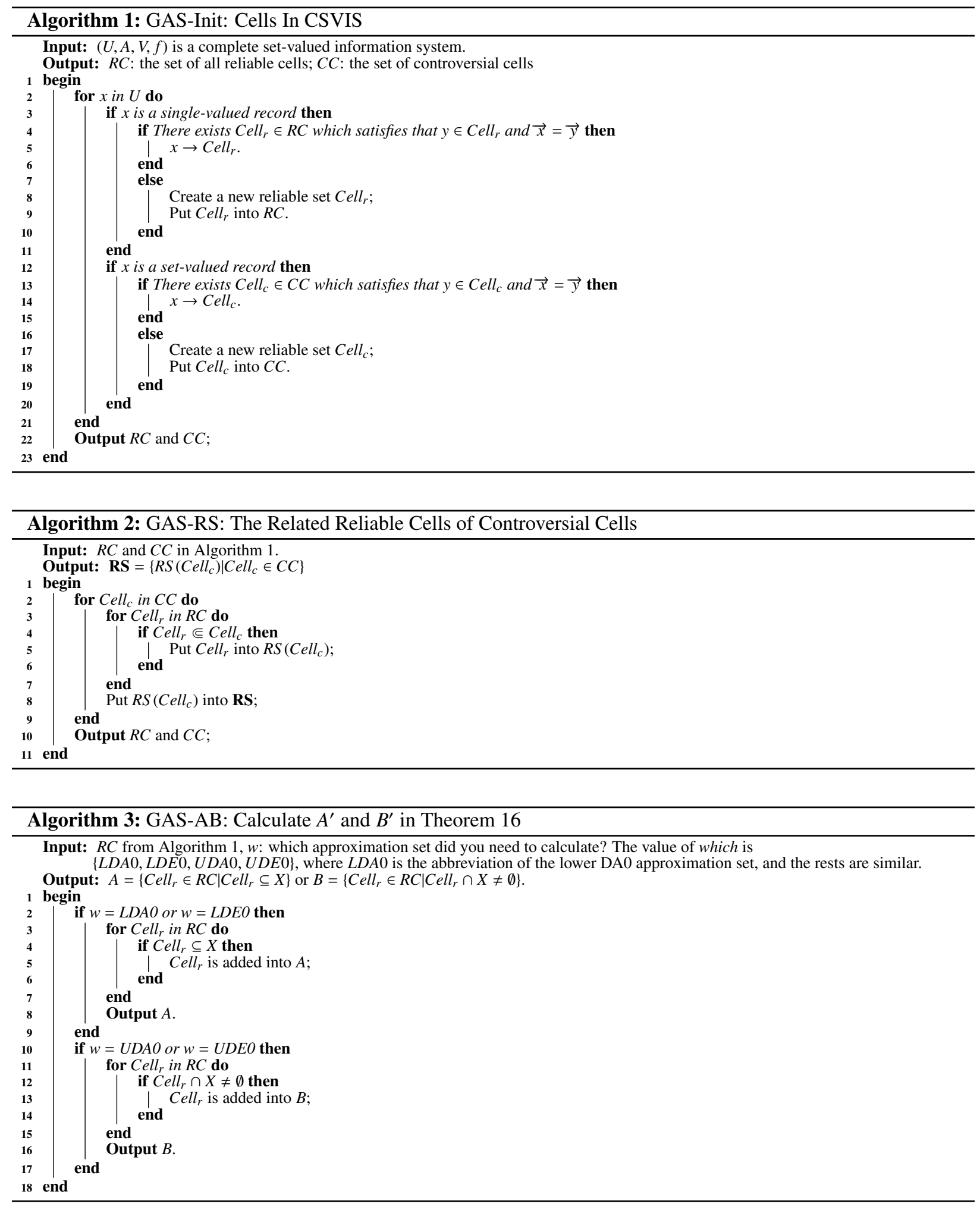


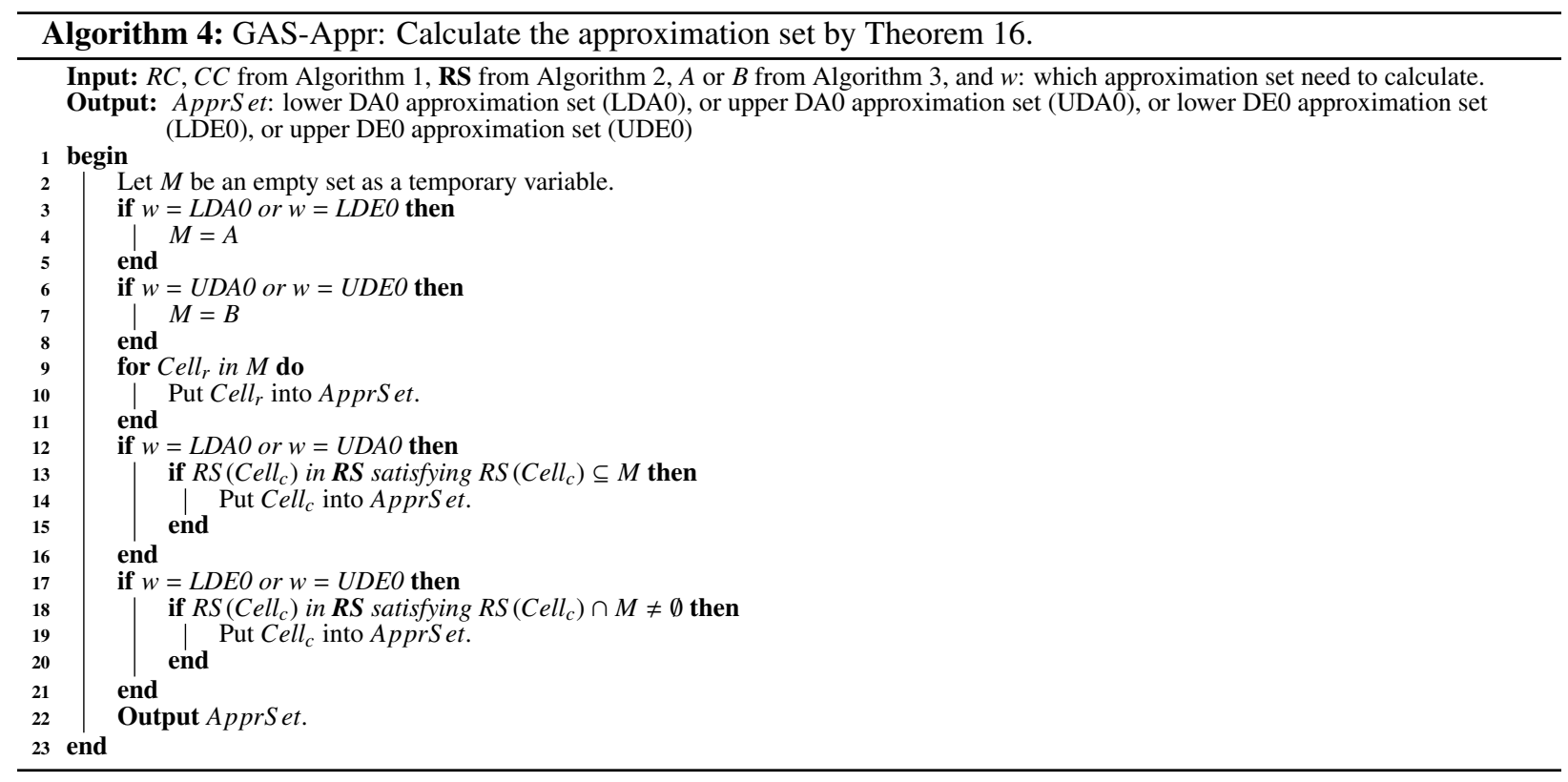

the GC0 approximation sets $A^{\prime}$ and $B^{\prime}$ have been calculated based on $X$ and $R C$ by Algorithm GAS-AB (Algorithm 4). The time complexity is $O(|R C|)$. When the preconditions of Theorem $16\left(C C, R C, R S, A^{\prime}\right.$, and $\left.B^{\prime}\right)$ are all ready, the approximation set of $X$ can be calculated in Algorithm GAS-Appr (Algorithm 4). Because $|R C| \geq\left|B^{\prime}\right| \geq\left|A^{\prime}\right|$ in general, the time complexity of GAS-Appr will $O(|R C|)+O(|C C|)$. In summary, the time complexity of Algorithm GAS is

$$
O(|U|+|C C| \times|R C|+|R C|+|R C|+|C C|) \approx O(|U|+|C C| \times|R C|) \approx \max (O(|U|, O(|C C| \times|R C|) .
$$

If $|C C|$ and $|R C|$ are less than $\sqrt{|U|}$, the time complexity of Algorithm GAS is $O(|U|)$, otherwise, the time complexity of Algorithm GAS would be $O(|C C| \times|R C|)$.

\section{Experimental Analysis}

We had downloaded 12 data sets from the machine learning data repository, University of California at Irvine (abbr. UCI) [? ] including two incomplete discrete value data sets ("Dress" and "House Votes") and another 10 complete continuous value data sets. All of them have been outlined in Table 2. What needs illustration is the "Cover Type" data set. To show the trend of calculation time of approximation sets on different scales of data sizes, we generated a serial of data sets based on "Cover Type" (more 500,000 records) —- CT-1, CT-2,..., CT-320. CT-X will contain one "X"th part of CT. For example, CT-320 contains a 320th part of the whole data set. And only 11 continuous condition attributes in "Cover Type" were selected in the generated data set.

All UCI data sets in Table 2 were transformed into set-valued information system $(U, A, V, f)$ as experimental data. For incomplete discrete-valued data sets ("Dress" and "House Votes"), the missing value is filled with the set of all possible attribute values - $V_{a}$ of $a \in A$. For another continuous-valued data sets, the attribute value will be into two intervals by the mean and median at the same time. The specific transformation from continuous value to set value is shown in Figure 5. If a value is labeled as "\{Small, Small\}", the value corresponds to a single value "Small"; If a value is labeled as " $\{\mathrm{Big}, \mathrm{Big}\}$ ", the value corresponds to another single value "Big"; If the labels of value are different, the value is controversial and it corresponds to set value $\{$ Small, Big\}. Furthermore, to keep the uniform of those universes, all set-valued information systems are complete. Therefore, as a pretreatment, we remove the set-valued records, in which the related single-valued cell set is empty. The size of CSVIS has been listed in the last column of Table 2. 
Table 2: Data Sets in Experiement

\begin{tabular}{lllll}
\hline Data Sets & Short Name & Samples & Attributes & Samples in CSVIS \\
\hline Anuran Calls & AC & 7195 & 22 & 6291 \\
Avila (Train Set) & AV & 10430 & 10 & 9947 \\
Congressional Voting & CV & 435 & 16 & 341 \\
Dresses & DR & 501 & 13 & 107 \\
Letter Recoginization & LR & 20000 & 16 & 15354 \\
Magic Gamma Telescope & MG & 19020 & 11 & 18835 \\
Page Blocks & PB & 5473 & 10 & 4697 \\
Pen Recoginization & PR & 10992 & 16 & 10443 \\
Wine & WI & 178 & 13 & 143 \\
Wine Quality - Red & WR & 1599 & 11 & 1416 \\
Wine Quality - White & WW & 4989 & 11 & 4775 \\
Cover Type & CT & 581012 & $54(11)$ & 576031 \\
\hline
\end{tabular}

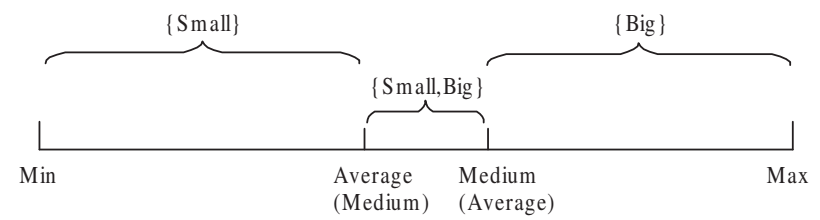

Figure 5: Transform a Continuous-Valued Data Set into a Set-Valued Information System

In this section, a comparative study was performed about approximation quality and calculation time among DEO and DA0 approximation sets, DA and DE approximation sets [? ], A-approximation set (MTA) and E-approximation set (MTE) based on maximal tolerance classes [? ], approximation sets based on tolerance relation (Tol) [? ? ? ], non-symmetric tolerance relation (NST) [? ? ], and limited tolerance relation (LNT) [? ]. The prefix "L" and "U" in the abbreviation of the approximation operator represents "Lower" and "Upper" respectively For example, "LNST" is the abbreviation of lower approximation sets based on non-symmetric tolerance classes.

These algorithms about DE0, DA0, and other tolerance relation approximation operators are coded in Java (JDK 8u_121 and Eclipse Neon 3). All experiments are conducted on a computer with a single Intel Xeon CPU E3 1231 V3 (4 cores, 3.4GHz), 16GB (DDR3 1600) RAM. The operating system is 64-bit Windows 7 Ultimate.

\subsection{Performance Comparison about Approximation Quality}

\subsubsection{Approximation Quality Comparison Experiment about Approximation Sets}

The approximation quality of approximation sets is the main standard to measure the uncertainty of rough set. The high approximation quality means that the approximation set should approach to approximated sets $X$ as closely as possible. A simple and common index, the dependency of approximation set $-\rho$, is adopted to measure the quality of approximation sets in this paper.

$$
\rho=\frac{\sum_{i=1}^{n}\left|A p p r\left(D_{i}\right)\right|}{\sum_{i=1}^{n}\left|D_{i}\right|}
$$

where $\operatorname{Appr}\left(D_{i}\right)$ is an approximation set and $D_{i}$ is a decision class in the set-valued information system. A high-quality approximation set means that $\rho$ should be as close to 1 as possible.

The approximation quality $\rho$ of DE0, DA0, DE, DA, MTA, MTE, Tol, NST, and LNT approximation sets are depicted in Figure 6, where x-coordinate pertains to the name of approximation operator, y-coordinate concerns the approximation quality $\rho$. To highlight DE0 and DA0 approximation sets, the points about them are solid, and the points about the comparative approximation operators are open. To show the details, we list the top 3 in approximation quality of those approximation sets in Table 3. As seen in Figure 6 and Table 3, DE0 and DA0 approximation sets (solid points) are generally closer to 1 than other approximation sets (open points) on 12 data sets. Meanwhile, the results in Figure 6 verify the equivalence between DE (DA) [? ] and MTE (MTA) [? ] in CSVIS. They appeared the same approximation quality in every data set. Therefore, in Table 3, we make no distinction between them. 


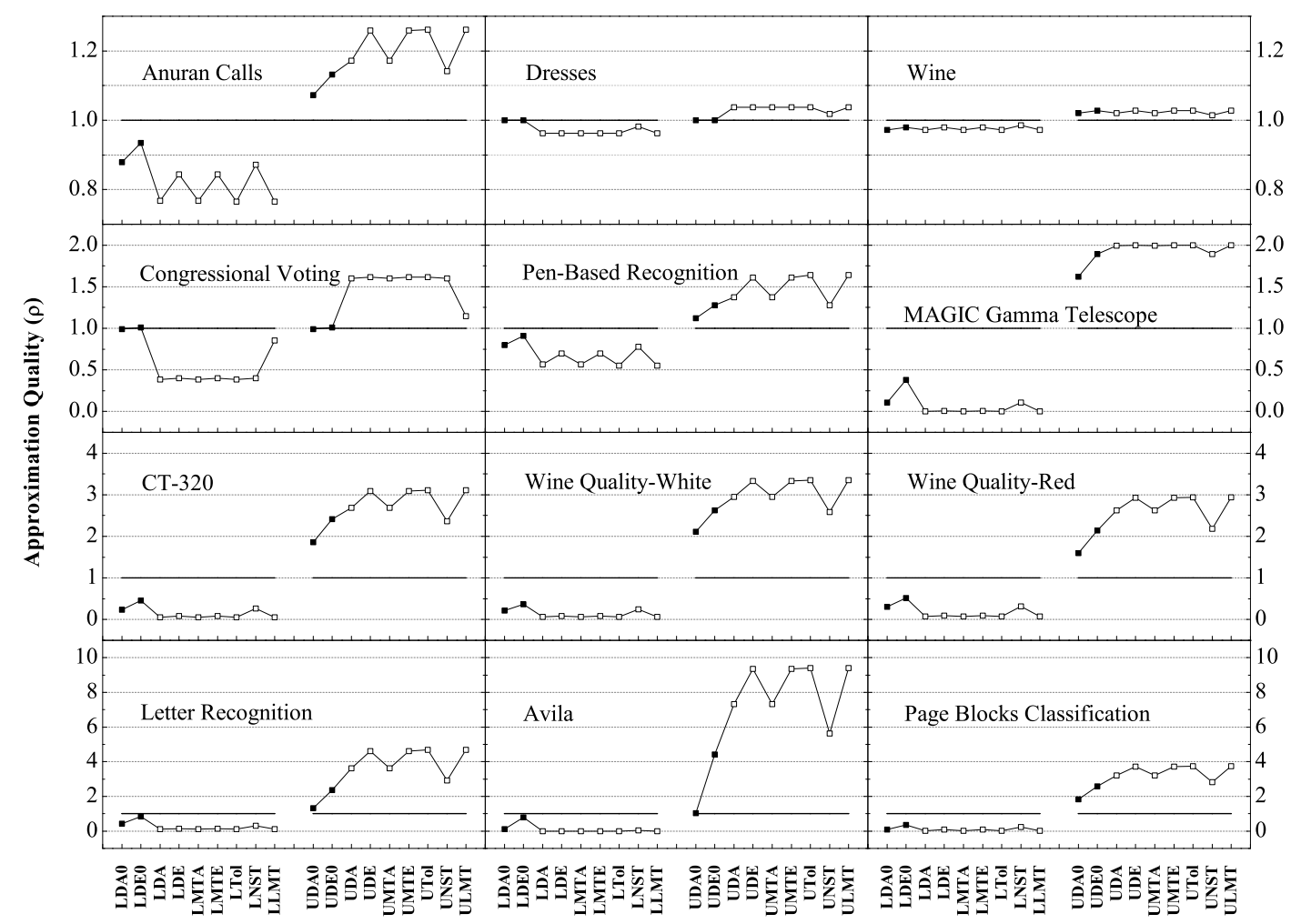

Figure 6: Approximation Quality of DE0, DA0, DE, DA, MTA, MTE, Tol, LNT, NST Approximation Sets

Table 3: Top 3 in Approximation Quality of Approximation Sets in Different Data Sets

\begin{tabular}{llll|lll}
\hline \multirow{2}{*}{ Data Name } & \multicolumn{2}{c}{ Appr. Quality $\rho$ of Lower Appr. Set } & \multicolumn{2}{c}{ Appr. Quality $\rho$ of Upper Appr. Set } \\
\cline { 2 - 7 } & Top 1 & Top 2 & Top 3 & Top 1 & Top 2 & Top3 \\
\hline AC & DE0 & DA0 & NST & DA0 & DE0 & NST \\
& $(0.934)$ & $(0.879)$ & $(0.872)$ & $(1.072)$ & $(1.132)$ & $(1.141)$ \\
AV & DE0 & DA0 & NST & DA0 & DE0 & NST \\
& $(0.787)$ & $(0.104)$ & $(0.037)$ & $(1.032)$ & $(4.411)$ & $(5.630)$ \\
CV & DE0 & DA0 & LMT & DA0 & DE0 & LMT \\
& $(1.011)$ & $(0.988)$ & $(0.853)$ & $(0.988)$ & $(1.011)$ & $(1.146)$ \\
CT-320 & DE0 & NST & DA0 & DA0 & NST & DE0 \\
& $(0.458)$ & $(0.265)$ & $(0.240)$ & $(1.863)$ & $(2.361)$ & $(2.413)$ \\
DR & DE0 & DA0 & NST & DA0 & DE0 & NST \\
& $(1.000)$ & $(1.000)$ & $(0.981)$ & $(1.000)$ & $(1.000)$ & $(1.018)$ \\
LR & DE0 & DA0 & NST & DA0 & DE0 & NST \\
& $(0.840)$ & $(0.425)$ & $(0.310)$ & $(1.316)$ & $(2.354)$ & $(2.917)$ \\
MG & DE0 & DA0 & NST & DA0 & DE0 & NST \\
& $(0.378)$ & $(0.1079)$ & $(0.1076)$ & $(1.681)$ & $(1.8920)$ & $(1.8923)$ \\
PB & DE0 & NST & DE $\backslash$ MTE & DA0 & DE0 & NST \\
& $(0.349)$ & $(0.223)$ & $(0.091)$ & $(1.832)$ & $(2.589)$ & $(2.821)$ \\
PR & DE0 & DA0 & NST & DA0 & DE0 & NST \\
& $(0.907)$ & $(0.796)$ & $(0.778)$ & $(1.120)$ & $(1.2785)$ & $(1.2786)$ \\
WI & NST & DE0 & DE $\backslash$ MTE & NST & DA0 & DA $\backslash$ MTA \\
& $(0.986)$ & $(0.979)$ & $(0.979)$ & $(1.013)$ & $(1.020)$ & $(1.020)$ \\
WR & DE0 & NST & DA0 & DA0 & DE0 & NST \\
& $(0.521)$ & $(0.314)$ & $(0.306)$ & $(1.598)$ & $(2.144)$ & $(2.177)$ \\
WW & DE0 & NST & DA0 & DA0 & NST & DE0 \\
& $(0.371)$ & $(0.247)$ & $(0.210)$ & $(2.112)$ & $(2.585)$ & $(2.622)$ \\
\hline
\end{tabular}




\subsubsection{Approximation Quality Comparison Experiment about Single-valued Records in Approximation Sets}

In general, those single-valued records in set-valued information systems are more valuable than the set-valued records. Thus, how much single-valued records are in approximation sets is another important fact to measure the approximation quality of approximation sets in set-valued information systems. DE and DE0 approximation sets had been employed as the representatives of those approximation sets in the comparative experiments. The reasons are as follows:

a) Wherever in the neighborhood based on tolerance relations or block in covering, two single-valued records in a neighborhood or block mean that their information explanations are the same. In another word, their corresponding attribute values of two single-valued records are equal.

b) Those approximation operators based on SMC, Tol, NST, and LNT have the same operator's form

$$
\underline{T}(X)=\{x \mid \operatorname{Tol}(x) \subseteq X\}, \bar{T}(X)=\{x \mid \operatorname{Tol}(x) \cap X \neq \emptyset\},
$$

where $\operatorname{Tol}(x)$ is the neighborhood of $x$ or the block which contains $x$. Based on the same approximation space, operator's form and basis algebra, the conclusion is reasonable that the projections of those approximation sets on $U_{0}$ are the same. For any $X \subseteq U$,

$$
\begin{gathered}
\underline{C}_{D A}\left(X \cap U_{0}\right) \cap U_{0}=\underline{C}_{D E}\left(X \cap U_{0}\right) \cap U_{0}=\underline{C}_{M T A}\left(X \cap U_{0}\right) \cap U_{0}=\underline{C}_{M T E}\left(X \cap U_{0}\right) \cap U_{0} \\
=\underline{C}_{T o l}\left(X \cap U_{0}\right) \cap U_{0}=\underline{C}_{N S T}\left(X \cap U_{0}\right) \cap U_{0}=\underline{C}_{L M T}\left(X \cap U_{0}\right) \cap U_{0} ; \\
\bar{C}_{D A}\left(X \cap U_{0}\right) \cap U_{0}=\bar{C}_{D E}\left(X \cap U_{0}\right) \cap U_{0}=\bar{C}_{M T A}\left(X \cap U_{0}\right) \cap U_{0}=\bar{C}_{M T E}\left(X \cap U_{0}\right) \cap U_{0} \\
=\bar{C}_{T o l}\left(X \cap U_{0}\right) \cap U_{0}=\bar{C}_{N S T}\left(X \cap U_{0}\right) \cap U_{0}=\bar{C}_{L M T}\left(X \cap U_{0}\right) \cap U_{0} .
\end{gathered}
$$

c) The single-valued records in DE0 and DA0 approximation sets are the same (Theorem 12-a and b).

$$
\underline{C}_{D A 0}(X) \cap U_{0}=\underline{C}_{D E 0}(X) \cap U_{0}, \bar{C}_{D A 0}(X) \cap U_{0}=\bar{C}_{D E 0}(X) \cap U_{0} .
$$

Therefore, the comparison experiments about the single-valued record in approximation sets between the reliable SMC approximation operators (DE0, DA0) and other approximation operators (DE, DA, MTA, MTE, Tol, NST, LMT) can be simplified as a comparison between DE0 and DE.
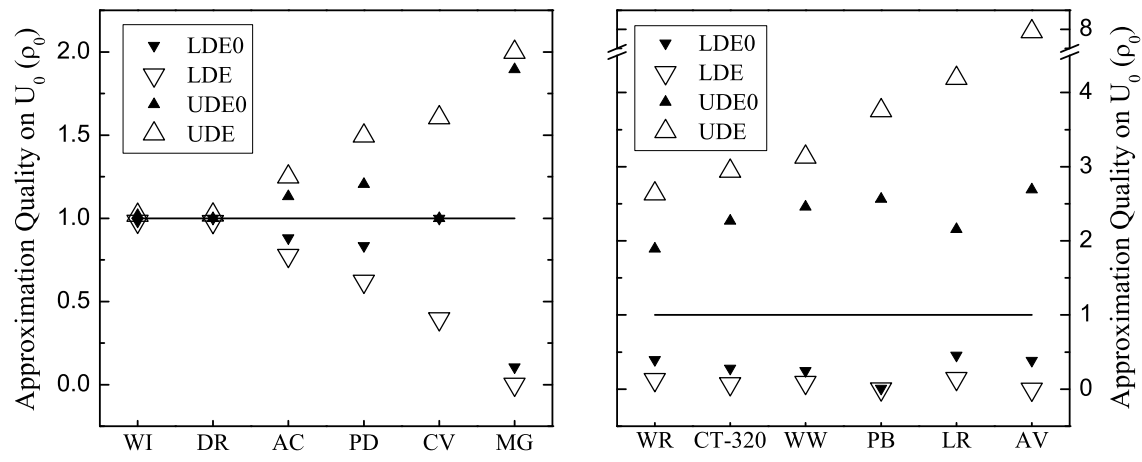

Figure 7: Approximation Quality about Single-valued Records $\rho_{0}$ of DE0, DA0, DE, DA, MTA, MTE, Tol, LNT, NST Approximation Sets

The comparative results are depicted in Figure 7. In Figure 7, the x-coordinated pertains to the data set name, and the point in the y-coordinate is the dependency $\rho_{0}$ of approximation set on $U_{0}$.

$$
\rho_{0}=\frac{\sum_{i=1}^{n}\left|\operatorname{Appr}\left(D_{i}\right) \cap U_{0}\right|}{\left|U_{0}\right|}
$$

where $\operatorname{Appr}\left(D_{i}\right)$ is a DE0 or DE approximation set and $D_{i}$ is the decision class in the set-valued information system. It is shown that lower (upper) DE0/DA0 approximation sets could contain (reject) more necessary (reductive) singlevalued records than the DE/DA, MTE/MTA, Tol, LMT, NST ones. Meanwhile, the results also match the conclusions in Corollary 7. 


\subsubsection{Approximation Quality Comparison Experiment about Weak Monotonicity in Approximation Sets}

In general, a lower approximation set should be in the approximated set, and the upper one should cover the approximated set in rough set theory. Unfortunately, because of the weak monotonicity, the DE0/DA0 approximation sets can not satisfy the restrictive condition. The essential reason comes from the original setting about controversial elements for DE0 and DA0 approximation sets. Whether a controversial element belongs to an approximation set or not totally depends on its related reliable cells. Therefore, some unconventional controversial elements appear, which can be considered as the cost of improvement in DE0 and DA0.
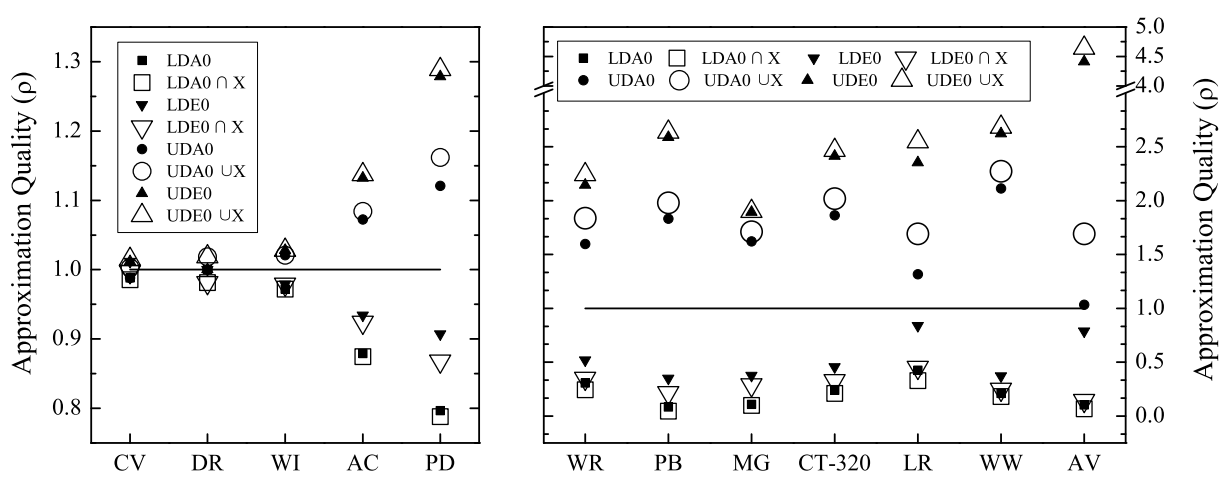

Figure 8: Approximation Quality about Weak Monotonicity $\rho$ of DE0, DA0, DE, DA, MTA, MTE, Tol, LNT, NST Approximation Sets

In Figure 8, the x-coordinate pertains to the dataset name, and the point in the y-coordinate is the dependency of the approximation set. The solid points are the dependency for DE0 and DA0 approximation sets in Figure 6. The open ones are the dependency of the modified DE0 and DA0 approximation sets. For the lower DE0 and DA0 approximation sets, we removed the elements out of $X$ and denoted them as "LDE $0 \cap X$ " and "LDA $0 \cap X$ "; For the upper DE0 and DA0 approximation sets, we attached the elements in $X$, and denoted them as "UDE0 $\cup \mathrm{X}$ " and "UDA0 $\cup X$ ". The distance between the solid point and the open one reflects the ratio of exceptional controversial elements in approximation sets in Figure 8. Meanwhile, we have also noticed that the exceptional controversial elements in DE0 and DA0 approximation sets are widespread. In most datasets, such as CV, DR, WI, AC, PD, WR, PB, MG, CT320 , WW, the ratio of those exceptional controversial elements is low, and the influence of exceptional controversial elements is so little as to be unnoticeable. But in other datasets, such as LR, AV, the influence is not negligible. If we use the rough approximation sets as a data filter to clean the original data set, the misjudgment of the exceptional controversial elements will deeply affect the precise of the following training model.

\subsection{Performance Comparison about Calculation Time}

Calculation time is another important fact in the actual application of approximation set specially for those large scale datasets. We performed a comparative study about the calculation performance of DE0, DA0, DE, DA, MTE, MTA, Tol, NST, and LMT approximation sets at the above data sets. For DE0 and DA0 approximation sets, we have employed granular algorithm GAS in section 5.4, which includes $4 \mathrm{sub}$ algorithms - GAS-Init, GAS-RS, GAS-AB and GAS-Appr (Algorithm 1 - Algorithm 4), to speed up the calculation process. For DE and DA approximation sets, we recalled the granular algorithms (G-DE and Cut-DA) from [? ]. For other approximation operators, we will calculate their approximation sets following their original definition. Specifically, we will construct a normal, maximal, non-symmetric, or limited tolerance classes $\operatorname{Tol}(x)$ for every element in the universe at first, and then calculate the Tol, MTA, MTE, NST, and LMT approximation sets based on the following approximation form.

$$
\underline{T}(X)=\{x \mid \operatorname{Tol}(x) \subseteq X\}, \bar{T}(X)=\{x \mid \operatorname{Tol}(x) \cap X \neq \emptyset\} .
$$

The comparative results are shown in Figure 9. In Figure 9, the x-coordinate pertains to the approximation operator, and a point in the y-coordinate is the calculation time of those corresponding approximation sets. It is shown that the 


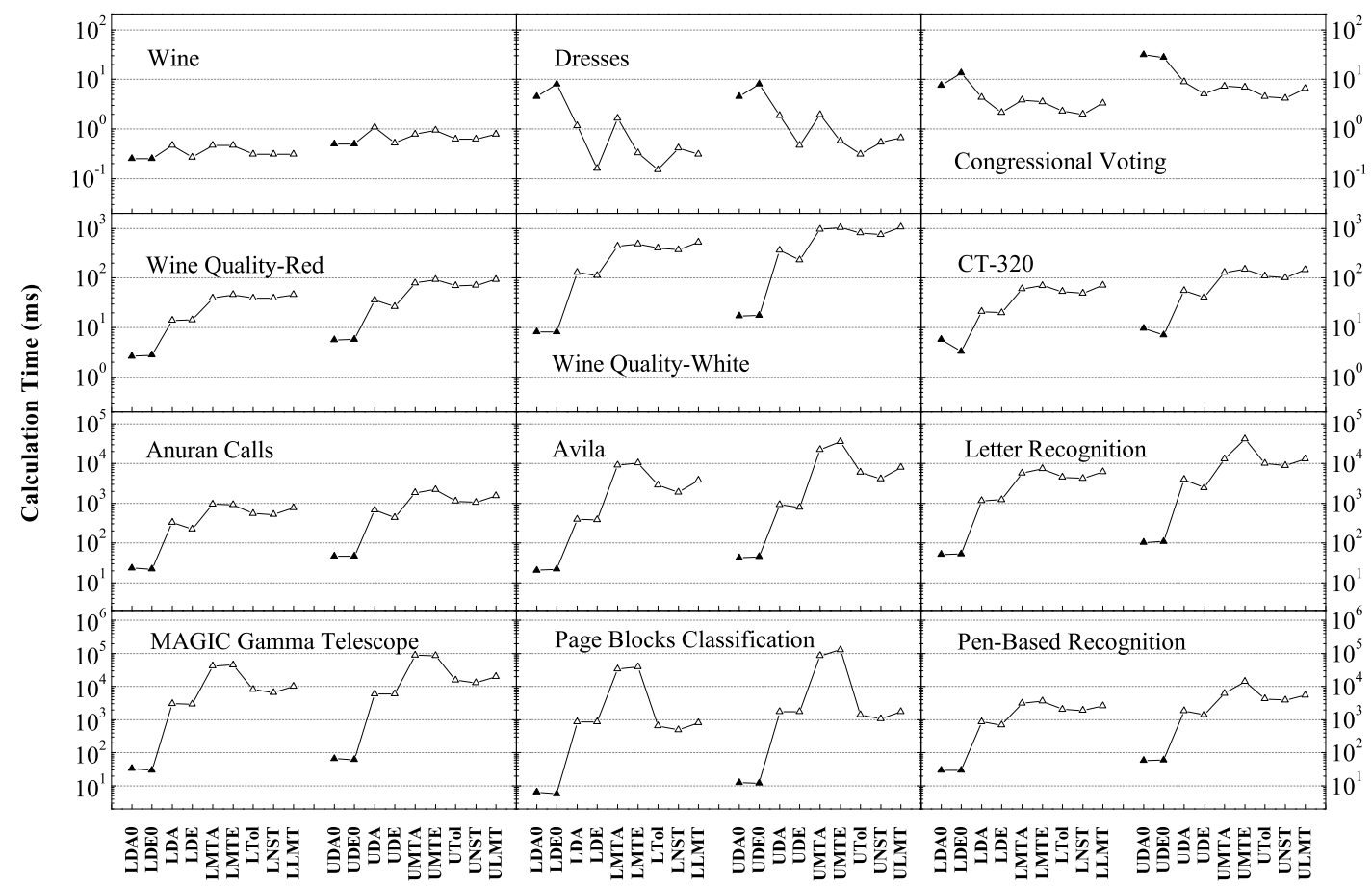

Figure 9: Calculation Time of DE0, DA0, DE, DA, MTA, MTE, Tol, LNT, NST Approximation Sets

calculation time of algorithm GAS is less than others at data sets while the size of data sets increases. Because of the long pipeline in Algorithm GAS, GAS took almost the same even more time to finish its job in the small data sets WI (173 records), DR (501 records), CV (435 records). While the size of data sets increase to 1,000+, the advantage of Algorithm GAS appears.

To evaluate the computational performance of those approximation sets when the data sizes increasing, we conduct compared experiments on a series of data sets (CT-1, CT-2, ...., CT-320). CT-1 contains the whole (581012) records in CT, CT-2 contains half of the records (290506) in CT, CT-5 contains one-fifth (116202) records in CT, ..., the last CT-320 contains one 320th (1815) records. As the limitation of the computer's memory, we can not perform the computation of MTA, MTE, Tol, LNT, and NST approximation sets at CT-5, and barely finished the jobs of DE and DA approximation sets on CT-2. Through DE and DA failed at CT-1, the granular algorithm of DE and DA still demonstrated excellent speedup performance comparing to MTE and MTA. Finally, granular algorithm GAS of DE0 and DA0 approximation sets finish the whole testing in an acceptable time and memory capacity. The comparative results show in Figure 10. In Figure 10, the x-coordinate is the names of data sets, and the y-coordinate concerns the sum of calculation time of lower and upper approximation sets. The calculation time of Algorithm GAS for DE0 and DA0 is far lower than others. When the data size increases, the advantage of Algorithm GAS is gradually expanding. In Figures 9 and 10, the computation time of Algorithm GAS is not only far less than other algorithms based on the original definition by granular computing but also less than the granular algorithm for DE and DA by reducing the repeated computing of controversial cells.

The series of experiments about approximation quality and calculation time indicate that DE0 and DA0 approximation sets on set-valued information systems are not only quality but also easy to compute. 


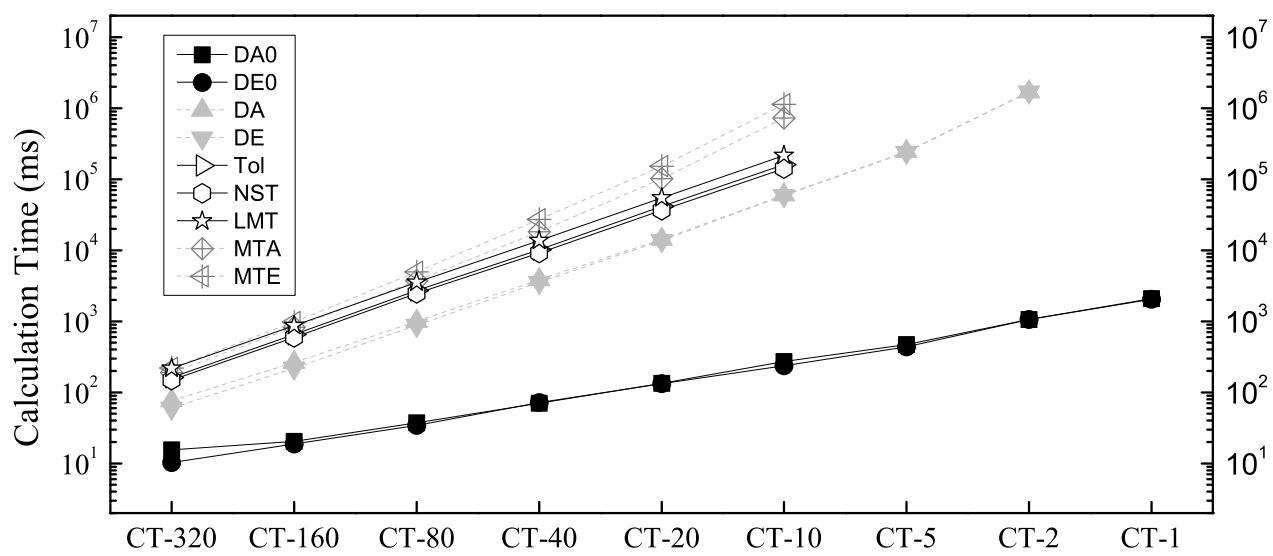

Figure 10: Calculation Time of DE0, DA0, DE, DA, MTA, MTE, Tol, LNT, NST Approximation Sets in CT Serial Data Sets

\section{Conclusion}

In summary, our contribution in this paper is to provide a high-quality and efficient computational rough set model for the complete set-valued information systems. The weak monotonicity of approximation sets and the requirement of completeness for set-valued information systems are the necessary compromises for the high approximation quality and efficient computation.

Semi-monolayer covering (SMC), as a special representative covering, is a new approach to organize the tolerance classes in set-valued (incomplete) information systems. Though existing DE and DA [? ] have granule-based form and easy to calculate, there is some room for improvement because the reliable and controversial elements had been allowed to equally participate in the calculation of the approximation sets. In this paper, we proposed two improved approximation operators based on semi-monolayer covering - DE0 and DA0. Apparently, in DE0 and DA0, the singlevalued records set (reliable set) replaced the whole block to participate in judging the relation of approximated set $X$ (Definition 8). Virtually, DE0 and DA0 have a piecewise design to distinguish reliable elements from controversial elements. The reliable sets in approximation have been firstly confirmed. The controversial elements only can be added according to the rules about the reliable sets (Theorem 14 and 16, Algorithm 1 - 4). The main performances of DE0 and DA0 are as follows:

- DE0 and DA0 approximation sets are more quality than DE and DA approximation sets, A-approximation and E-approximation based on maximal tolerance classes, the approximation sets based on tolerance classes, limit tolerance classes, and non-symmetric tolerance classes in many real data sets. Theorem 6, Corollary 7, and the experiments about approximation quality in section 5.1 (Figure 6 and 7) confirmed the advantage of DE0 and DA0 in approximation quality wherever in theory and application.

- DE0 and DA0 have the equivalent granule-based form based on the information cell in set-valued information systems (Theorem 16). Using the granular algorithm (Algorithm 3 and 4), DE0 and DA0 approximation sets can be calculated more rapidly than other element-based form approximation sets based on tolerance classes, even than the granule-based form DE and DA approximation sets (Figure 9). While the data set increases, the growth rate of the calculating time of algorithm GAS is much lower than other models (Figure 10). It shows us the potential to develop the DE0 and DA0 approximation sets into parallel computing and big data [? ? ].

Nothing is perfect. The improved operator's form in DE0 and DA0 has a little negative effect caused by controversial elements. DE0 and DA0 approximation sets only satisfy the monotonicity on $U_{0}$ (Theorem 11 ) rather than on $U$ (Theorem 10). It means that there exist some exceptional controversial elements in DE0 and DA0 approximation 
sets which may break the monotonicity between the approximation sets and the approximated set. Although we can confirm that the exceptional controversial elements are some minor set-valued records in set-valued information systems (Theorem 11), the example under Theorem 10 and the experiment about approximation quality (Section 6.1.3, Figure 8) show that the exceptional controversial elements are widespread. In some cases, weak monotonicity maybe becomes the Achilles' heel for the application of DE0 and DA0 approximation sets. It is a necessary and meaningful job to predict and control the overflowed controversial elements in approximated sets.

\section{Acknowledgements}

This work was supported by the National Natural Science Foundation of China (11601129, 61972134).

\section{References}

[1] Waseem Ahmed, M. M. Sufyan Beg, and Tanvir Ahmad. Entropy based feature selection for fuzzy set-valued information systems. 3d Research, 9(2):19, 2018.

[2] Zbigniew Bonikowski. Algebraic structures of rough sets in representative approximation spaces. Electronic Notes in Theoretical Computer Science, 82(4):52-63, 2003.

[3] Zbigniew Bonikowski, Edward Bryniarski, and Urszula Wybraniec-Skardowska. Extensions and intentions in the rough set theory. Information Sciences, 107(1-4):149-167, 1998.

[4] Yi Cheng. The incremental method for fast computing the rough fuzzy approximations. Data and Knowledge Engineering, 70:84-100, 2011.

[5] Ines Couso and Didier Dubois. Rough sets, coverings and incomplete information. Fundamenta Informaticae, 21:1001-1025, 2001.

[6] Jianhua Dai and Haowei Tian. Entropy measures and granularity measures for set-valued information systems. Information Sciences, 240:72$82,2013$.

[7] Jianhua Dai and Haowei Tian. Fuzzy rough set model for set-valued data. Fuzzy Sets and Systems, 229(oct.16):54-68, 2013.

[8] Dheeru Dua and Casey Graff. UCI machine learning repository, 2017.

[9] Didier Dubois and Henri Prade. Rough fuzzy sets and fuzzy rough sets. International Journal of General Systems, 17:191-209, 1990.

[10] Salvatore Greco, Benedetto Matarazzo, and Roman Slowinski. Rough sets theory for multicriteria decision analysis. European Journal of Operational Research, 129(1):1-47, 2001.

[11] Yan Yong Guan and Hong-Kai Wang. Set-valued information systems. Information Sciences, 176(17):2507-2525, 2006.

[12] Yanting Guo, Eric C.C. Tsang, Meng Hu, Xuxin Lin, Degang Chen, Weihua Xu, and Binbin Sang. Incremental updating approximations for double-quantitative decision-theoretic rough sets with the variation of objects. Knowledge-Based Systems, 189:105082, 2020.

[13] Chengxiang Hu, Shixi Liu, and Xiaoling Huang. Dynamic updating approximations in multigranulation rough sets while refining or coarsening attribute values. Knowledge-Based Systems, 130:62-73, 2017.

[14] Chengxiang Hu, Shixi Liu, and Guoxiu Liu. Matrix-based approaches for dynamic updating approximations inmultigranulation rough sets. Knowledge-Based Systems, 122:51-69, 2017.

[15] Jun Hu and Guoyin Wang. Uncertainty problem processing with covering generalized rough sets. Intelligent Systems Reference Library, 43:293-308, 2013.

[16] Marzena Kryszkiewicz. Rough set approach to incomplete information systems. Information Sciences, 112(1-4):39-49, 1998.

[17] Marzena Kryszkiewicz. Rules in incomplete information systems. Information Sciences, 113(3-4):271-292, 1999.

[18] Yee Leung and Deyu Li. Maximal consistent block technique for rule acquisition in incomplete information systems. Information Sciences, 153:85-106, 2003.

[19] Yee Leung, Wei-Zhi Wu, and Wen-Xiu Zhang. Knowledge acquisition in incomplete information systems: A rough set approach. European Journal of Operational Research, 168(1):164-180, 2006

[20] Shaoyong Li, Zhiyong Hong, and Tianrui Li. Efficient composing rough approximations for distributed data. Knowledge-Based Systems, $182: 104793,2019$.

[21] Shaoyong Li and Tianrui Li. Incremental update of approximations in dominance-based rough sets approach under the variation of attribute values. Information Sciences, 294:348-361, 2015.

[22] Shaoyong Li, Tianrui Li, Zhixue Zhang, Hongmei Chen, and Junbo Zhang. Parallel computing of approximations in dominance-based rough sets approach. Knowledge-Based Systems, 87:102 - 111, 2015. Computational Intelligence Applications for Data Science.

[23] Tsau Young Lin, Yiyu Yao, and Lotfi A. Zadeh, editors. Data Mining, Rough Sets and Granular Computing. Springer-Verlag, Berlin Heidelberg, 2002.

[24] W Lipski. On databases with incomplete information. Journal of the Acm, 28(1):41-70, 1981.

[25] Dun Liu, Tianrui Li, and Junbo Zhang. Incremental updating approximations in probabilistic rough sets underthe variation of attributes. Knowledge-Based Systems, 73:81-96, 2015.

[26] Y. Liu and W. Zhu. On three types of covering-based rough sets via definable sets. In IEEE International Conference on Fuzzy Systems, pages 1226-1233, 2014.

[27] Chuan Luo, Tianrui Li, Hongmei Chen, and Lixia Lu. Fast algorithms for computing rough approximations inset-valued decision systems while updating criteria values. Information Sciences, 299:221-242, 2015.

[28] Chuan Luo, Tianrui Li, and Yiyu Yao. Dynamic probabilistic rough sets with incomplete data. Information Sciences, 417:39-54, 2017.

[29] John N. Mordeson. Rough set theory applied to (fuzzy) ideal theory. Fuzzy Sets and Systems, 121(2):315-324, 2001.

[30] Do Van Nguyen, Koichi Yamada, and Muneyuki Unehara. Extended tolerance relation to define a new rough set model in incomplete information systems. Advances in Fuzzy Systems, 2013:1-10, 2013. 
[31] Ewa Orlowska and Zdzislaw Pawlak. Representation of nondeterministic information. Theoretical Computer Science, 29(1-2):27-39, 1987.

[32] Zdzislaw Pawlak. Rough sets. International Journal of Computer and Information, 11:341-356, 1982.

[33] Janusz Andrzej Pomykala. Approximation operations in approximation space. Bulletin of the Polish Academy of Sciences Mathematics, 35(1):653-662, 1987.

[34] Yuhua Qian, Xinyan Liang, Qi Wang, Jiye Liang, Bing Liu, Andrzej Skowron, Yiyu Yao, Jianmin Ma, and Chuangyin Dang. Local rough set: A solution to rough data analysis in big data. International Journal of Approximate Reasoning, 97:38 - 63, 2018.

[35] Keyun Qin, Yan Gao, and Zheng Pei. On covering rough sets. In Rough Set and Knowledge Technology, pages 34-41, 2007.

[36] Anna Maria Radzikowskaa and Etienne E. Kerre. A comparative study offuzzy rough sets. Fuzzy Sets and Systems, 126:137-155, 2002.

[37] Muhammad Summair Raza and Usman Qamar. A parallel approach to calculate lower and upper approximations in dominance based rough set theory. Applied Soft Computing, 84:105699, 2019.

[38] Jerzy Stefanowski and Alexis Tsoukias. Incomplete information tables and rough classification. Computational Intelligence, 17(3):545-566, 2001.

[39] Fei Yue Wang and William Zhu. The fourth type of covering-based rough sets. Information Sciences, 201:80-92, 2012.

[40] Guoyin Wang. Extension of rough set under incomplete information systems. In IEEE International Conference on Fuzzy Systems, pages 1098-1103, 2002.

[41] Qi Wang, Yuhua Qian, Xinyan Liang, Qian Guo, and Jiye Liang. Local neighborhood rough set. Knowledge-Based Systems, 153:53 - 64, 2018.

[42] Shu Wang, Tianrui Li, Chuan Luo, and Hamido Fujita. Efficient updating rough approximations with multi-dimensional variation of ordered data. Information Sciences, 372:690 - 708, 2016.

[43] Shu Wang, Tianrui Li, Chuan Luo, Jie Hu, Hamido Fujita, and Tianqiang Huang. A novel approach for efficient updating approximations in dynamic ordered information systems. Information Sciences, 507:197 - 219, 2020.

[44] Wei Wei, Junbiao Cui, Jiye Liang, and Junhong Wang. Fuzzy rough approximations for set-valued data. Information Sciences, 360:181-201, 2016.

[45] Chen Wu and Xibei Yang. Information granules in general and complete coverings. In 2005 IEEE International Conference on Granular Computing, volume 2, pages 675-678 Vol. 2, 2005.

[46] Wei Zhi Wu, You Hong Xu, Ming Wen Shao, and Guo Yin Wang. Axiomatic characterizations of (s, t)-fuzzy rough approximation operators. Information Sciences, 334-335:17-43, 2016.

[47] Wei Zhi Wu and Wen-Xiu Zhang. Constructive and axiomatic approaches of fuzzy approximation operators. Information Sciences, 159(34):233-254, 2004.

[48] Zhengjiang Wu, Ning Chen, and Yan Gao. Semi-monolayer cover rough set: Concept, property and granular algorithm. Information Sciences, 456:97-112, 2018.

[49] Zhengjiang Wu, Keyun Qin, Tianrui Li, and Ruan Da. Approximation operators, binary relation and basis algebra in l-fuzzy rough sets. Fundamenta Informaticae, 111(1):47-63, 2011.

[50] Zhengjiang Wu and Wenpeng Xu. Binary relation, basis algebra, approximation form and its property in l-fuzzy rough sets. Journal of Computers, 6(7):1501-1510, 2011.

[51] Xiaoliang Xie, Zhaowen Li, Pengfei Zhang, and Gangqiang Zhang. Information structures and uncertainty measures in an incomplete probabilistic set-valued information system. IEEE Access, 7:27501-27514, 2019.

[52] Xibei Yang, Zhang Ming, Huili Dou, and Jingyu Yang. Neighborhood systems-based rough sets in incomplete information system. Knowledge-Based Systems, 24(6):858-867, 2011.

[53] Xibei Yang, Yong Qi, Hualong Yu, and Jingyu Yang. Updating multigranulation rough approximations with increasingof granular structures. Knowledge-Based Systems, 64:59-69, 2014.

[54] Yiyu Yao and Yanhong She. Rough set models in multigranulation spaces. Information Sciences, 327:40-56, 2016.

[55] Yiyu Yao and Bingxue Yao. Covering based rough set approximations. Information Sciences, 200:91-107, 2012.

[56] Anping Zeng, Tianrui Li, Jie Hu, Hongmei Chen, and Chuan Luo. Dynamical updating fuzzy rough approximations for hybrid data under the variation of attribute values. Information Sciences, 378:363-388, 2016.

[57] Chucai Zhang and Jianhua Dai. An incremental attribute reduction approach based on knowledge granularity for incomplete decision systems. Granular Computing, pages https://doi.org/10.1007/s41066-019-00173-7, 2019.

[58] Junbo Zhang, Tianrui Li, Da Ruan, Zizhe Gao, and Chengbing Zhao. A parallel method for computing rough set approximations. Information Sciences, 194(none):209-223, 2012.

[59] Junbo Zhang, Tianrui Li, Da Ruan, and Dun Liu. Rough sets based matrix approaches with dynamic attribute variation in set-valued information systems. International Journal of Approximate Reasoning, 53(4):620-635, 2012.

[60] Junbo Zhang, Jian Syuan Wong, Tianrui Li, and Pan Yi. A comparison of parallel large-scale knowledge acquisition using rough set theory on different mapreduce runtime systems. International Journal of Approximate Reasoning, 55(3):896-907, 2014.

[61] Junbo Zhang, Yun Zhu, Yi Pan, and Tianrui Li. Efficient parallel boolean matrix based algorithms for computing composite rough set approximations. Information Sciences, 329:287 - 302, 2016. Special issue on Discovery Science.

[62] Yingying Zhang, Tianrui Li, Chuan Luo, Junbo Zhang, and Hongmei Chen. Incremental updating of rough approximations in interval-valued information systems under attribute generalization. Information Sciences, 373:461 - 475, 2016.

[63] William Zhu and Fei Yue Wang. Relationships among three types of covering rough sets. In 2006 IEEE International Conference on Granular Computing. DBLP, 2006

[64] Wojciech Ziarko. Variable precision rough set model. Journal of Computer and System Science, 46(1):39-59, 1993.

[65] Wojciech Ziarko. Probabilistic approach to rough sets. International Journal of Approximate Reasoning, 49(2):272-284, 2008. 